\title{
Triviality properties of principal bundles on singular curves
}

\author{
Prakash Belkale and Najmuddin Fakhruddin
}

\begin{abstract}
We show that principal bundles for a semisimple group on an arbitrary affine curve over an algebraically closed field are trivial provided that the order of the $\pi_{1}$ of the group is invertible in the ground field or the curve has semi-normal singularities. Several consequences and extensions of this result (and method) are given. As an application, we realize conformal blocks bundles on moduli stacks of stable curves as push-forwards of line bundles on (relative) moduli stacks of principal bundles on the universal curve.
\end{abstract}

\section{Introduction}

It is a consequence of a theorem of Harder [Har67, Satz 3.3] that generically trivial principal $G$-bundles on a smooth affine curve $C$ over an arbitrary field $k$ are trivial if $G$ is a semisimple and simply connected algebraic group. When $k$ is algebraically closed and $G$ reductive, generic triviality, conjectured by Serre, was proved by Steinberg [Ste65] and Borel-Springer [BS68].

It follows that principal bundles for simply connected semisimple groups over smooth affine curves over algebraically closed fields are trivial. This fact (and a generalization to families of bundles [DS95]) plays an important role in the geometric realization of conformal blocks for smooth curves as global sections of line bundles on moduli stacks of principal bundles on the curves (see the overview [Sor96] and the references therein).

An earlier result of Serre [Ser58, Théorème 1] (see also [Ati57, Theorem 2]) implies that this triviality property is true if $G=\mathrm{SL}(r)$ and $C$ is a possibly singular affine curve over an arbitrary field $k$. In [BG18], it was shown by a versal deformation argument that if $X$ is a reduced projective curve with at worst nodal singularities over $\mathbb{C}$, with $p_{1}, \ldots, p_{n}$ in the smooth locus of $X$ such that $\mathcal{O}\left(\sum p_{i}\right)$ is ample, there exists a dense open substack of the moduli of $G$-bundles on $X$ such that any $E$ in this open substack restricts to a trivial bundle on $X \backslash\left\{p_{1}, \ldots, p_{n}\right\}$.

The results of this paper show that the triviality properties of principal bundles on arbitrary singular curves (in particular, degenerating families of smooth curves) are very similar to those on smooth curves. They allow us to realize conformal blocks on moduli stacks of stable curves $\overline{\mathcal{M}}_{g, n}$ as push-forwards of line bundles on (relative) moduli stacks of principal bundles on the universal curve (Theorem 1.7).

Received 24 September 2016, accepted in final form 5 December 2017.

2010 Mathematics Subject Classification 14D20, 14H60, 14H20.

Keywords: principal bundles, singular curves, $B$-reduction, Drinfeld-Simpson theorem, conformal blocks.

This journal is (C) Foundation Compositio Mathematica 2019. This article is distributed with Open Access under the terms of the Creative Commons Attribution Non-Commercial License, which permits non-commercial reuse, distribution, and reproduction in any medium, provided that the original work is properly cited. For commercial re-use, please contact the Foundation Compositio Mathematica. 


\section{PRINCIPAL BUNDLES ON SINGULAR CURVES}

Alternate compactifications of $\mathcal{M}_{g, n}$ have been considered recently (cf. the Hassett-Keel program [FS13]); triviality statements for $G$-bundles over arbitrary affine curves could potentially be useful in a geometric theory of conformal blocks over such spaces.

Theorem 1.1. Let $C$ be an arbitrary (possibly non-reduced, reducible or disconnected) affine curve over an algebraically closed field $k$. If $G$ is a semisimple algebraic group over $k$ such that $\left|\pi_{1}(G)\right|$ is invertible in $k$, then any principal $G$-bundle $E$ on $C$ is trivial; that is, there is a section $s: C \rightarrow E$.

The main idea is, assuming $C$ to be reduced, to produce a section over the normalization of $C$ (using [Har67]) which on the inverse image of a (suitable) infinitesimal neighborhood $C_{m}$ of the singular locus of $C$ agrees with the pull-back of a section of $E$ over $C_{m}$. Such sections are shown to descend to $C$.

The conditions for triviality in Theorem 1.1 are necessary and sufficient for arbitrary singularities, but for semi-normal curves a stronger result holds; see Section 3.3.

ThEOREM 1.2. Let $C$ be an arbitrary separated curve over an algebraically closed field $k$ and $E$ a principal $G$-bundle on $C$ for a connected reductive group $G$ over $k$.

(a) Let $Z$ be a finite subset of $C$. Then, there exists a Zariski-open subset $U$ of $C$ containing $Z$ such that $E$ is trivial on $U$.

(b) The structure group of $E$ can be reduced to $B$, where $B$ is a Borel subgroup of $G$.

Note that in the case of generically reduced $C$, Theorem 1.2 follows easily from Theorem 1.1 if the order of $\pi_{1}$ of the semisimple quotient of $G$ is invertible in $k$, but there are no conditions on the characteristic of $k$ or on $C$ in Theorem 1.2.

A result of Białynicki-Birula [Bia70, Theorem 1] implies that if $C$ is irreducible, then any $E$ as in Theorem 1.2 is Zariski-locally trivial. However, Zariski-local triviality does not seem to imply the existence of $B$-structures (that is, that the structure group of $E$ can be reduced to $B$ ) if the curve has more than one singular point.

We also prove versions of Theorems 1.1 and 1.2 which allow for families of non-constant curves (Theorems 1.3, 1.4 and 1.5 below). Using Theorem 1.2(b) as input, these follow, very closely, arguments of Drinfeld and Simpson [DS95] for similar results for families of smooth curves.

Let $S$ be an arbitrary scheme over $\operatorname{Spec}(\mathbb{Z})$, and let $f: X \rightarrow S$ be a proper, flat and finitely presented curve over $S$. Let $G$ be a split ${ }^{1}$ reductive group scheme over $\operatorname{Spec}(\mathbb{Z})$ (base changed to $S$ ) and $B$ a Borel subgroup of $G$.

Theorem 1.3. Let $E$ be a principal $G$-bundle on $X$ with $G$ connected and reductive. Then, after a surjective étale base change $S^{\prime} \rightarrow S$, the structure group of $E$ can be reduced to $B$ (and hence $E$ becomes Zariski-locally trivial).

Let $D \subset X$ be a relatively ample effective Cartier divisor which is flat over $S$, and let $U=X \backslash D$.

TheOrem 1.4. Let $E$ be a principal $G$-bundle on $X$ with $G$ semisimple and simply connected. Then, after a surjective étale base change $S^{\prime} \rightarrow S$, the bundle $E$ is trivial on $U_{S^{\prime}}$.

\footnotetext{
${ }^{1}$ As is well known, any reductive group scheme becomes split after a surjective étale base change, and so the splitness assumption is not required in Theorems 1.3, 1.4 and 1.5.
} 


\section{P. Belkale And N. FAKhruddin}

For $G=\mathrm{SL}(n)$, Zariski localization is sufficient in Theorem 1.4 in many cases; see Remark 3.4.

If $G$ is not simply connected, we need further hypotheses, having to do with the existence of relative Picard schemes, reductions to $\mathrm{Pic}^{0}$ and the nature of the $\mathrm{Pic}^{0}$ of fibers, while generalizing arguments in [DS95].

Theorem 1.5. Let $f: X \rightarrow S$ be a proper, flat and finitely presented curve, with $f$ cohomologically flat in dimension zero (see [BLR90, Section 9.4, p. 259]), $D \subset X$ a relatively ample and flat (over $S$ ) effective Cartier divisor and $E$ a principal $G$-bundle on $X$. Assume that

(A) étale locally on $S$, the divisor $D \subset X$ is, set theoretically, a union of (possibly not disjoint) sections of $f$;

(B) the morphism $f$ is smooth in a neighborhood of $D$;

(C) the group $G$ is semisimple, with $\left|\pi_{1}(G)\right|$ invertible in $\mathcal{O}_{S}$.

Then, after a surjective étale base change $S^{\prime} \rightarrow S$, the bundle $E$ is trivial on $U_{S^{\prime}}$, where $U=X \backslash D$.

Note that the cohomological flatness condition on $f$ holds if it has reduced geometric fibers. We discuss a variant of this theorem in Section 3.3.

The methods used in Theorem 1.1 can be used in the study of questions related to the Grothendieck-Serre conjecture.

THEOREM 1.6. Let $X$ be a reduced surface over an algebraically closed field whose normalization is smooth. Let $G$ be a connected reductive group and $E$ a principal $G$-bundle on $X$ which is generically trivial. Then $E$ is locally trivial in the Zariski topology.

Note that there exist examples of principal bundles over normal surfaces which are generically trivial but not locally trivial; see Section 4.1. Over non-algebraically closed fields, there exist such bundles even over (singular) curves [AG60].

In Section 5, we prove the irreducibility of the moduli stack of $G$-bundles on a singular projective curve when $G$ is semisimple and simply connected (Proposition 5.1), an extension of the uniformization theorem for G-bundles [BL94, BL95, LS97, DS95] for singular curves (Proposition 5.2) and the integrality of the space of maps from a reduced affine curve to $G$ when the base field is $\mathbb{C}$ and the group $G$ is semisimple and simply connected (Proposition 5.3). The proof of Proposition 5.3 uses Theorem 1.4 and follows closely a proof of a similar result by Laszlo and Sorger [LS97]. A new group-theoretic input here is work on subgroups of split simply connected semisimple groups generated by elementary matrices [IM65, Ste73] over semilocal rings.

Recall that associated to a simple Lie algebra $\mathfrak{g}$ over $k=\mathbb{C}$ and dominant integral weights $\lambda_{1}, \ldots, \lambda_{n}$ at a level $\ell$ (see Section 6.1 ), the theory of conformal blocks produces vector bundles of conformal blocks $\mathbb{V}_{\mathfrak{g}, \lambda, \ell}$ on the moduli stacks of stable $n$-pointed curves $\overline{\mathcal{M}}_{g, n}$. Work in the 1990s due to several authors [BL94, Fal94, KNR94] led to a realization of the duals of fibers of $\mathbb{V}_{\mathfrak{g}, \lambda, \ell}$ over $\mathcal{M}_{g, n}$ as global sections of line bundles over suitable moduli spaces and stacks. Using Theorem 1.4 and the work of Beauville, Laszlo and Sorger (see the overviews [Sor96, Sor00]), we extend the stack-theoretic realization of conformal blocks to all of $\overline{\mathcal{M}}_{g, n}$ : Let $G$ be a simple, simply connected, complex algebraic group with Lie algebra $\mathfrak{g}$. In Section 6 , we consider a relative smooth Artin stack of parabolic bundles

$$
\pi: \operatorname{Parbun}_{G, g, n} \rightarrow \overline{\mathcal{M}}_{g, n},
$$

construct a line bundle $\mathcal{L}$ on $\operatorname{Parbun}_{G, g, n}$, and obtain the following result. 


\section{PRINCIPAL BUNDLES ON SINGULAR CURVES}

TheOREM 1.7. There is a canonical isomorphism $\pi_{*} \mathcal{L} \stackrel{\sim}{\rightarrow} \mathbb{V}_{\mathfrak{g}, \lambda, \ell}^{*}$

Such isomorphisms are produced for any family of stable $n$-pointed curves. A proof of the above statement for a fixed singular stable pointed curve also appears in [BG18] (which uses a different method).

For classical groups (and even levels in the case of Spin groups), the line bundle $\mathcal{L}$ can be constructed explicitly in terms of the determinant of cohomology (see Theorem 6.9). Finally, Picard groups of moduli stacks of parabolic bundles on (arbitarily) singular projective curves are computed in Section 7.

\section{Proof of Theorems 1.1 and 1.2}

We will first prove Theorem 1.1 under the assumption that $C$ is reduced and then prove the case of arbitrary $C$; until further notice, we assume that $C$ is reduced. Let $\pi: \widetilde{C} \rightarrow C$ be its normalization, let $G$ be a semisimple algebraic group and $E$ a principal $G$-bundle on $C$. We assume that $\left|\pi_{1}(G)\right|$ is invertible in $k$.

Let $R \subset C$ be a finite reduced subscheme such that $\pi$ is an isomorphism over $C \backslash R$. Let $C_{m}$ be the $m$ th infinitesimal neighborhood of $R$ in $C$, for $m \geqslant 0$. Let $\widetilde{C}_{m}=\pi^{-1}\left(C_{m}\right)$.

Lemma 2.1. There exists an $m \geqslant 0$ with the following property: $f \in H^{0}\left(\widetilde{C}, \mathcal{O}_{\widetilde{C}}\right)$ is in the image of $H^{0}\left(C, \mathcal{O}_{C}\right)$ if and only if the restriction of $f$ in $H^{0}\left(\widetilde{C}_{m}, \mathcal{O}_{\widetilde{C}_{m}}\right)$ is in the image of $H^{0}\left(C, \mathcal{O}_{C}\right)$ (equivalently, $H^{0}\left(C_{m}, \mathcal{O}_{C_{m}}\right)$ ).

Proof. The quotient $M=H^{0}\left(\widetilde{C}, \mathcal{O}_{\widetilde{C}}\right) / H^{0}\left(C, \mathcal{O}_{C}\right)$ is a finite $A$-module supported on $R$ for $A=$ $H^{0}\left(C, \mathcal{O}_{C}\right)$ and is hence annihilated by a power $I^{m}$ of the ideal $I$ of $R$ in $C$; hence, $M \stackrel{\sim}{\rightarrow}$ $M \otimes A / I^{m}$. Tensor the exact sequence

$$
0 \rightarrow H^{0}\left(C, \mathcal{O}_{C}\right) \rightarrow H^{0}\left(\widetilde{C}, \mathcal{O}_{\widetilde{C}}\right) \rightarrow M \rightarrow 0
$$

by $A / I^{m}$ to get the right-exact sequence

$$
H^{0}\left(C, \mathcal{O}_{C_{m}}\right) \rightarrow H^{0}\left(\widetilde{C}, \mathcal{O}_{\widetilde{C}_{m}}\right) \rightarrow M \otimes A / I^{m} \rightarrow 0
$$

which implies the desired assertion.

Fix $m$ as in Lemma 2.1. Suppose that $E$ is a principal $G$-bundle on $C$. Let $\widetilde{E}$ be the pull-back G-bundle on $\widetilde{C}$.

Lemma 2.2. Any section $s_{m}$ of $\widetilde{E}$ over $\widetilde{C}_{m}$ extends to a section of $\widetilde{E}$ over $\widetilde{C}$.

Proof. Since $\widetilde{E}$ is trivial as a $G$-bundle by [Har67] (see Remark 2.4), we only need to show that any $\gamma_{m}: \widetilde{C}_{m} \rightarrow G$ extends to a map $\gamma: \widetilde{C} \rightarrow G$, which is Lemma 2.5 below.

Lemma 2.3. Suppose that a section $s$ of $\widetilde{E}$ on $\widetilde{C}$ when restricted to $\widetilde{C}_{m}$ is the pull-back of a section of $E$ on $C_{m}$. Then $s$ is the pull-back of a section of $E$ on $C$.

Proof. Composing by the natural map $\widetilde{E} \rightarrow E$, we have a map $s^{\prime}: \widetilde{C} \rightarrow E$ which when restricted to $\widetilde{C}_{m}$ is a composition $\widetilde{C}_{m} \rightarrow C_{m} \stackrel{\alpha_{m}}{\rightarrow} E$. We need to show that $s^{\prime}$ is itself a composition $\widetilde{C} \rightarrow C \stackrel{\alpha}{\rightarrow} E$. This assertion is local on $E$ (we may find an affine open subset of $E$ which contains the image of $\widetilde{C}_{m}$ ). Arguing coordinate-by-coordinate using Lemma 2.1, we see that $s^{\prime}$ descends to a map $C \rightarrow E$, since the restrictions to $\widetilde{C}_{m}$ come from functions on $C_{m}$ (and any relation between coordinate functions which holds on $\widetilde{C}$ holds on $C$ as well since functions on $C$ embed into functions on $\widetilde{C}$ ). 


\section{P. Belkale And N. FAKhruddin}

Proof of Theorem 1.1 in the case $C$ reduced. There is a section of $E$ over the reduced scheme $R$ (defined above) since $R$ consists of finitely many points and $k$ is algebraically closed. Since $E \rightarrow C$ is smooth and $C_{m} \supset R$ is a nilpotent extension of $R$, there exists a section $C_{m} \rightarrow E$ over $C_{m}$ extending this section. Let $s_{m}$ be the induced section of $\widetilde{E}$ over $\widetilde{C}_{m}$. We extend $s_{m}$ to a section $s$ of $\widetilde{E}$ over $\widetilde{C}$ using Lemma 2.2. By Lemma 2.3, this section descends to $C$. Therefore, $E$ has a section over $C$ and is hence trivial.

Remark 2.4. Harder [Har67] assumes that $G$ is simply connected and therefore any principal $G$-bundle on a smooth affine curve (over an algebraically closed field) is trivial if $G$ is semisimple and simply connected. If $G$ is not simply connected but still semisimple, let $\widetilde{G}$ be the simply connected cover of $G$. If $\left|\pi_{1}(G)\right|$ is invertible in $k$, then $\tau: \widetilde{G} \rightarrow G$ is étale with finite kernel, and the map $H_{\mathrm{et}}^{1}(C, \widetilde{G}) \rightarrow H_{\mathrm{et}}^{1}(C, G)$ is surjective (since $H_{\mathrm{et}}^{2}(C, \operatorname{ker}(\tau))=0$ ). Therefore, any principal $G$-bundle on $C$ comes from a principal $\widetilde{G}$-bundle and hence is trivial on $C$ (this reasoning is contained in [Har67, Satz 3.3]).

Lemma 2.5. Suppose that $\left|\pi_{1}(G)\right|$ is invertible in $k$. Any morphism $\gamma_{m}: \widetilde{C}_{m} \rightarrow G$ extends to a morphism $\gamma: \widetilde{C} \rightarrow G$.

Proof. Let $\widetilde{G}$ be the simply connected form of $G$. Since $\left|\pi_{1}(G)\right|$ is invertible in $k$, the morphism $\widetilde{G} \rightarrow G$ is étale. Thus, since $k$ is algebraically closed, by Lemma 2.6 (applied to $\widetilde{G}$ and to the residue fields of all points in $\widetilde{C}_{m}$, to get a morphism of $k$-schemes $\left(\widetilde{C}_{m}\right)_{\text {red }} \rightarrow V$; further liftings of $\left(\widetilde{C}_{m}\right) \rightarrow V$ are constructed out of smoothness of $\left.V \rightarrow G\right)$ and the infinitesimal lifting property for smooth morphisms, there exists a morphism $\tau: \mathbb{A}_{k}^{n} \rightarrow G$ such that $\gamma_{m}$ lifts to a morphism $\gamma_{m}^{\prime}: \widetilde{C}_{m} \rightarrow \mathbb{A}_{k}^{n}$.

By Lemma 2.7 below, we can extend $\gamma_{m}^{\prime}$ to a map $\gamma^{\prime}: \widetilde{C} \rightarrow \mathbb{A}_{k}^{n}$. The desired $\gamma$ is then $\tau \circ \gamma^{\prime}$.

Lemma 2.6. Let $k$ be an arbitrary field and $G$ a simply connected, semisimple and split group over $k$. There exist a morphism $\eta: \mathbb{A}_{k}^{n} \rightarrow G$ and an open subset $V \subset \mathbb{A}_{k}^{n}$ such that $\left.\eta\right|_{V}$ is smooth and for any extension $K$ of $k$, the map $V(K) \rightarrow G(K)$ induced by $\eta$ is surjective.

Proof. Choose a collection of one-parameter unipotent subgroups $U_{i} \stackrel{\sim}{\rightarrow} \mathbb{G}_{a} \subseteq \widetilde{G}$, for $i=1, \ldots, s$, whose tangent spaces at the origin $\operatorname{span}^{2}$ the Lie algebra of $\widetilde{G}$. Consider the multiplication map

$$
\eta: U:=U_{1} \times_{k} U_{2} \times_{k} \cdots \times_{k} U_{s} \rightarrow \widetilde{G} \rightarrow G .
$$

By construction, $\eta$ is surjective on tangent spaces at the identity $e$ of $U$.

Since $G$ is simply connected, it is well known (see, for example, [Ste68]) that there exist $U^{\prime}$, a product of one-parameter unipotent groups as above, and $\eta^{\prime}: U^{\prime} \rightarrow G$ such that the induced map $U^{\prime}(K) \rightarrow G(K)$ is surjective for all $K / k$. Then $U \times U^{\prime} \stackrel{\sim}{\rightarrow} \mathbb{A}_{k}^{n}$ for some $n$ and the map $U \times U^{\prime} \rightarrow G$ given by $\eta \cdot \eta^{\prime}$ has the desired properties: if $\eta^{\prime}(x)=y$ for $x \in U^{\prime}(K)$, then $\eta(e) \eta^{\prime}(x)=y$ and $\eta \cdot \eta^{\prime}$ is smooth at $(e, x)$.

Lemma 2.7. Let $T \subseteq X$ be a closed subscheme of an affine scheme $X$ over an arbitrary field $k$ and $f: T \rightarrow \mathbb{A}_{k}^{n}$ a morphism. Then $f$ extends to a morphism $X \rightarrow \mathbb{A}_{k}^{n}$.

Proof. Write $T$ as the spectrum of $A / I$ with $I$ an ideal of $A=H^{0}\left(X, \mathcal{O}_{X}\right)$. The function $f$ corresponds to an $n$-tuple $\left(f_{1}, \ldots, f_{n}\right)$ of elements of $A / I$, which can be lifted to an $n$-tuple of elements in $A$.

\footnotetext{
${ }^{2}$ Simple coroots are in the span: this follows from the (easy) case of SL(2).
} 


\section{PRINCIPAL BUNDLES ON SINGULAR CURVES}

Remark 2.8. If $k$ is a finite field or the function field of a curve over an algebraically closed field, then any principal $G$-bundle on a curve over $k$ is generically trivial if $G$ is simply connected, semisimple and split [Har75, dJHS11]. Since Lemma 2.6 holds over an arbitrary field and principal $G$-bundles over $\operatorname{Spec}(k)$, for $k$ as above, are also trivial, the above proof of Theorem 1.1 also shows that principal $G$-bundles on affine curves over such fields are trivial if $G$ is simply connected, semisimple and split. (This also holds for most groups $G$ as above if $k$ has characteristic zero and is of cohomological dimension one (for example, a $C_{1}$-field): the function field of any curve over $k$ is perfect of cohomological dimension two, and a conjecture of Serre, known for all classical groups as well as some exceptional groups (see [BP95]), implies that any principal $G$-bundle (with $G$ as above) on a curve over $k$ is generically trivial.)

Proof of Theorem 1.1, general case. If $C$ is not reduced, let $C_{\text {red }} \subseteq C$ be the reduced subscheme. Let $E^{\prime}$ be the pull-back principal $G$-bundle on $C_{\text {red }}$. By the case of Theorem 1.1 for reduced curves proved above, $E^{\prime}$ is trivial, and hence we obtain a section $C_{\text {red }} \rightarrow E^{\prime}$. Composing with the natural map $E^{\prime} \rightarrow E$, we obtain a map $C_{\text {red }} \rightarrow E$ which when composed with $E \rightarrow C$ gives the natural inclusion $C_{\text {red }} \subseteq C$.

Since $E \rightarrow C$ is smooth, $C$ is affine and $C_{\text {red }} \subseteq C$ is given by a nilpotent ideal, by the infinitesimal criterion for smoothness, we can lift $C_{\text {red }} \rightarrow E$ (over $C_{\text {red }} \rightarrow C$ ) to a map $C \rightarrow E$ such that the composite $C \rightarrow E \rightarrow C$ is the identity map, that is, $s$ is a section, and hence $E$ is trivial.

\subsection{Proof of Theorem 1.2}

Since $B$-bundles are trivial in suitable Zariski neighborhoods of any given finite subset, statement (a) follows from statement (b). Note that it suffices to prove statement (a) when $C$ is reduced (trivializations on $U_{\text {red }}$ extend to $U$ when $U$ is affine).

2.1.1 For statement (b), we start with the case where $C$ is affine. We need to produce a section of $E / B$ over $C$. Since $E / B$ is smooth over $C$, we may assume that $C$ is reduced and are allowed to shrink $C$ to suitable affine neighborhoods of $R$ (since $E / B$ is projective over $C$ ). Since $\widetilde{E}$ has a $B$-structure, it is semilocally trivial. Now, replace $\widetilde{C}$ by an affine neighborhood containing $\pi^{-1}(R)$ (and $C$ by its image under $\pi$ ) over which $\widetilde{E}$ is trivial. Therefore, we may assume that $\widetilde{E} / B$ is the trivial bundle over $\widetilde{C}$ with fibers $G / B$.

We now replace $E$ by $E / B$ and $G$ by $G / B$ throughout in the proof of Theorem 1.1. Here, we note that there is a $B$-structure on $E$ restricted to any $C_{m}$, since $E / B$ is smooth over $C$. We then need to prove (the analog of Lemma 2.5) that any function $\bar{f}: \widetilde{C}_{m} \rightarrow G / B$ extends to a function $\widetilde{C} \rightarrow G / B$. This is easy because $G / B$ is covered by affine spaces (that is, of the form $\mathbb{A}_{k}^{m}$ ): we can use the group $G$ to move the image of $\bar{f}$ into an affine space and then apply Lemma 2.7.

2.1.2 Clearly, the case of affine $C$ implies statement (b) for all reduced $C$ (actually, all generically reduced $C$ ), because we can find an open affine subset of our curve which contains all singularities and a $B$-reduction of $E$ on this open set, and then extend using the valuative criterion for properness.

2.1.3 For the general case of statement (b) (that is, $C$ possibly not generically reduced), since we know that $E$ is generically trivial, we may extend $E$ to a compactification of $C$. Therefore, assume that $C$ is projective. By the case of reduced curves already considered above, we know 


\section{P. BELKAle AND N. FAKHRUdDin}

that $E$ has a $B$-reduction on $Z=C_{\text {red }}$. We now use methods from [DS95].

Definition 2.9. Let $\bar{\alpha}: B \rightarrow \mathbb{G}_{m}$ be the morphism associated to a positive root $\alpha$ of $G$. If $E_{B}$ is a principal $B$-bundle on $C$, let $E_{\alpha}$ be the line bundle on $C$ induced by $\bar{\alpha}$.

Using Lemma 2.11, choose a $B$-reduction of $E$ on $Z$ such that for every positive root $\alpha$, the line bundle $E_{\alpha}$ has sufficiently large degrees (to be made clear below) on irreducible components of $Z$. We consider the problem of lifting this $B$-reduction through nilpotent thickenings with square zero:

- Let $Z=C_{\text {red }} \subset C_{1} \subset C_{2} \subset C$ be nilpotent thickenings such that the ideal $\mathcal{I}$ of $C_{1}$ in $C_{2}$ has square zero.

- Also assume that we have lifted our $B$-reduction on $E$ over $Z$ to a $B$-reduction $\sigma_{1}: C_{1} \rightarrow$ $E / B$ over $C_{1}$.

Let $i_{1}: C_{1} \rightarrow C$ be the inclusion and $\Theta$ the relative tangent bundle of $E / B$ over $C$. The obstruction for lifting the reduction $\sigma_{1}$ further to $C_{2}$ lies in $H^{1}\left(C_{1}, \sigma_{1}^{*} \Theta \otimes \mathcal{I}\right)$. Since we can filter $\sigma_{1}^{*} \Theta$ by line bundles $i_{1}^{*} E_{\alpha}$, we are reduced to showing that $H^{1}\left(C_{1}, i_{1}^{*} E_{\alpha} \otimes \mathcal{I}\right)=0$ for all positive roots $\alpha$. This is true because of the following (applied to $X=C_{1}$ ), and therefore we can extend the $B$-reduction all the way to $C$, as desired.

Lemma 2.10. Let $\mathcal{F}$ be a coherent sheaf on a projective curve $X$ over an algebraically closed field. There is a positive integer $N=N(\mathcal{F})$ such that if $Z=X_{\text {red }}, Z=\cup Z_{i}$ with irreducible components $Z_{i}$ and $\mathcal{L}$ is a line bundle on $X$, then

$$
\operatorname{deg}_{Z_{i}}\left(\left.\mathcal{L}\right|_{Z_{i}}\right)>N, i=1, \ldots, s, \Longrightarrow H^{1}(X, \mathcal{L} \otimes \mathcal{F})=0 .
$$

Proof. Let $\mathcal{J}$ be the ideal of $Z$ in $X$. We may filter $\mathcal{F}$ by sheaves $\mathcal{J}^{s} \mathcal{F} / \mathcal{J}^{s+1} \mathcal{F}$ and may hence assume that $\mathcal{F}$ is a coherent sheaf on $Z$. Therefore, we reduce to the case where $X$ is reduced, which is standard.

LEMma 2.11. Let $E$ be a principal $G$-bundle on a reduced projective curve $Y$ over an algebraically closed field. Assume that $E$ has a $B$-reduction. Then, $E$ has a $B$-reduction such that for every positive root $\alpha$, the degree of the corresponding $E_{\alpha}$ on each irreducible component of $Y$ is at least $N$.

Proof. Each step in the proof of [DS95, Proposition 3] generalizes:

(i) By Theorem 1.2(a) in the case $C$ reduced, we can find an open affine $U \subset Y$ that contain all singular points of $C$ such that $E$ is trivial on $U$. We may now replace $E$ by any other $E^{\prime}$ which agrees with $E$ on $U$ (in other words, is trivial on $U$ ) provided that we change $N$ to a suitable $N^{\prime}$ (there is a bijection between $B$-reductions of $E$ and $E^{\prime}$ with bounded differences of degrees of $E_{\alpha}$ and $E_{\alpha}^{\prime}$ ).

(ii) We can therefore assume that $E$ is trivial.

(iii) Choose a finite morphism $Y \rightarrow \mathbb{P}^{1}$.

(iv) We are now reduced to the case $Y=\mathbb{P}^{1}$, which is the same as in [DS95].

In fact, we have shown the following result.

Proposition 2.12. Let $C$ be a projective curve over $k$ and $E$ a principal $G$-bundle. Then $E$ has a $B$-reduction $\sigma: C \rightarrow E / B$ such that $H^{1}\left(C, \sigma^{*} \Theta\right)=0$, where $\Theta$ is the relative tangent bundle of $E / B$ over $C$. 


\section{PRINCIPAL BUNDLES ON SINGULAR CURVES}

Proof. By Lemma 2.10, it would suffice if $E_{\alpha}$ had sufficiently large degrees on irreducible components of $C_{\text {red }}$. This follows from Lemma 2.11 and the construction above which extends a $B$ reduction on $C_{\text {red }}$ with sufficiently large degrees on irreducible components to one on $C$.

\section{Proofs of Theorems $1.3,1.4$ and 1.5}

In these proofs, we follow [DS95]: using Theorem 1.2(b) as basic input, the arguments of [DS95] carry over with obvious modifications.

\subsection{Proof of Theorem 1.3}

Let $F$ be the moduli functor of unobstructed $B$-reductions of $E$ : for a scheme $T$ over $S$, the space $F(T)$ is the space of $B$-reductions of $E \times{ }_{S} T$ over $X \times_{S} T$ such that for all $t \in T$,

$$
H^{1}\left(X_{t}, \sigma^{*} \Theta\right)=0,
$$

where $\Theta$ is the relative tangent bundle of $E / B$ over $X$ and $\sigma: X_{t} \rightarrow X$.

It follows from the theory of Hilbert schemes and deformation theory that $F$ is representable by a scheme $\phi: M \rightarrow S$ with $\phi$ smooth. To prove Theorem 1.3, it suffices to show that any $s \in S$ is in $\phi(M)$. This is because, by the smoothness of $\phi$, for any $s \in \phi(M)$, we can find an étale neighborhood $S^{\prime} \rightarrow S$ of $s$ such that there is a section $S^{\prime} \rightarrow M$ (over $S$ ).

To see that $s \in \phi(M)$, we may base change to the algebraic closure $k$ of $k(s)$. By Theorem 1.2(b) and Proposition 2.12, there is a $B$-reduction on $X_{s} \times_{k(s)} k$ with the desired vanishing property (3.1).

\subsection{Proof of Theorems 1.4 and 1.5}

We will prove Theorem 1.5 by following the proof of [DS95, Theorem 3]. The proof of Theorem 1.4 is similar, except that we do not need the "reduction to simply connected $G$ " part. In the following proof, $S$ is always assumed to be affine, and hence $U$ is also affine.

By Theorem 1.3, we find a $B$-reduction of $E$ after passing to an étale cover. Since $U$ is affine, the structure group of a $B$-bundle can be reduced to a maximal torus $H$ : in fact, if $E$ is a $B$ bundle on $X$ and $E_{H}$ the corresponding $H$-bundle, then $E$ and the $B$-bundle induced from $H$ via $H \rightarrow B$ are isomorphic over $U$. Therefore, we can assume that our principal bundle $E$ on $X$ comes from an $H$-bundle. The final step is then to show that any $H$-bundle on $X$ becomes trivial on $U$ after extension of the structure group to $G$ (and étale base change).

3.2.1 Reduction to the case of simply connected $G$. The arguments in [DS95, Section 6, second paragraph] for passage from $G$ to its simply connected cover $\widetilde{G}$ can be broken up into two

parts. The first is reduction to $\mathrm{Pic}^{0}$. For this, we note that cohomological flatness in dimension 0 implies, by a theorem of Artin, that $\mathrm{Pic}_{X / S}$ exists as an algebraic space over $S$ [Art69, Theorem 7.3], [BLR90, Section 8.3, Theorem 1]. Since any algebraic space has an étale covering by a scheme, this does not create any extra difficulty. The main idea in the reduction is then to modify an $H$-bundle on $X$, keeping it unchanged over $U$, so that for every $\tau \in A=\operatorname{Hom}\left(H, \mathbb{G}_{m}\right)$, the induced line bundle is in $\mathrm{Pic}^{0}$ of each fiber. Assumptions (A) and (B) in Theorem 1.5 allow us to make such a modification. This is because they imply that given any line bundle $L$ on $X$, we may, after an étale base change, find a Cartier divisor $D^{\prime}$ on $X$ with support contained in the support of $D$ such that $L\left(D^{\prime}\right)$ has degree 0 on each irreducible component of each fiber. 


\section{P. BELKAle AND N. FAKhruddin}

The second part concerns the natural homomorphism $T: \operatorname{Hom}\left(\widetilde{A}, \operatorname{Pic}^{0}\right) \rightarrow \operatorname{Hom}\left(A, \operatorname{Pic}^{0}\right)$, where $\widetilde{A}=\operatorname{Hom}\left(\widetilde{H}, \mathbb{G}_{m}\right)$ (here, $\widetilde{H}$ is the maximal torus of $\widetilde{G}$ over $H$ ). Since $A$ is a subgroup of $\widetilde{A}$ of index $\left|\pi_{1}(G)\right|$, the homomorphism $T$ is a morphism of group schemes over $S$ which, if the arithmetic genus of the fibers is positive, is étale if and only if $\left|\pi_{1}(G)\right|$ is invertible in $\mathcal{O}_{S}$.

Now, given a $G$-bundle $E$ on $X$, using Theorem 1.4, we may assume that it comes from a $B$-bundle $F$. On $U$, the bundle $E$ is isomorphic to the bundle induced from $F$ via the maps $B \rightarrow H \rightarrow G$, so we may assume that $E$ is induced from an $H$-bundle $E^{\prime}$, and we obtain a section of $\operatorname{Hom}\left(A, \mathrm{Pic}^{0}\right)$ over $S$. Form the fiber product $S^{\prime}$ of $S$ and $\operatorname{Hom}\left(\widetilde{A}, \mathrm{Pic}^{0}\right)$ over $\operatorname{Hom}\left(A, \operatorname{Pic}^{0}\right)$. Clearly, $S^{\prime} \rightarrow S$ is étale and surjective, and the pull-back of $E^{\prime}$ to $X \times_{S} S^{\prime}$ can be lifted to a $\widetilde{H}$-bundle locally with respect to the Zariski topology of $S^{\prime}$. We may therefore assume that $E$ is induced from a $\widetilde{G}$-bundle and comes from a $\widetilde{H}$-bundle.

Remark 3.1. If $\left|\pi_{1}(G)\right|$ is not invertible in $\mathcal{O}_{S}$, then $T$ is flat if (and only if) the $\mathrm{Pic}^{0}$ of the geometric fibers are semi-abelian.

3.2.2 The case of simply connected $G$. Now, assume that $G$ is simply connected and that $E$ comes from an $H$-bundle. Since $G$ is simply connected, $\operatorname{Hom}\left(\mathbb{G}_{m}, H\right)$ is freely generated by simple coroots. Therefore, we are reduced to checking that if $H$-bundles $E_{1}$ and $E_{2}$ differ by the image of some $\mathbb{G}_{m}$-bundle via a coroot $\check{\alpha}: \mathbb{G}_{m} \rightarrow H$, then the $G$-bundles corresponding to $E_{1}$ and $E_{2}$ are isomorphic on $U_{S}$ after Zariski localization in $S$.

The following simple lemma is used without proof in [DS95, Section 6]; we give one here for the reader's convenience.

Lemma 3.2. Let $L \subseteq G$ be the subgroup generated by $H$ and $r(\mathrm{SL}(2))$, where $r: \mathrm{SL}(2) \rightarrow G$ corresponds to $\alpha$ (so $\check{\alpha}$ factors through $r$ as in [Con14, Theorem 1.2.7, Definition 1.2.8]). Then $L=\mathrm{SL}(2) \times T$ or $L=\mathrm{GL}(2) \times T^{\prime}$, where $T$ and $T^{\prime}$ are subtori of $H$. Furthermore, $\tilde{r}$, which is $r$ viewed as a map $\mathrm{SL}(2) \rightarrow L$, is given by $\tilde{r}(g)=g \times e$ in both cases.

Proof. We first note that $r$ is an isomorphism onto its image since $G$ is simply connected; we use this to identify $r(\mathrm{SL}(2))$ with $\mathrm{SL}(2)$.

Let $Z$ be the identity component of the (reduced) center of $L$, so $Z$ is a codimension one subtorus of $H$. The inclusions induce a surjective morphism

$$
\pi: \operatorname{SL}(2) \times Z \rightarrow L .
$$

Let $K$ be the (scheme-theoretic) intersection of $Z$ and $\mathrm{SL}(2)$. The (scheme-theoretic) kernel of $\pi$ is then equal to the image of $K$ embedded diagonally in SL(2) $\times Z$.

Thus, if $K$ is trivial, then $\pi$ is an isomorphism, so we get the first possibility (take $T=Z$ ). Otherwise, we have $K \cong \mu_{2}$ since it is contained in the (scheme-theoretic) center of SL(2) and is non-trivial. Write $Z$ as a product $A \times T^{\prime}$, where $A \cong \mathbb{G}_{m}$ contains $K$; that this is always possible is easily seen using character groups. The subgroup $M$ of $L$ generated by $A$ and $\mathrm{SL}(2)$ is isomorphic to $\mathrm{GL}(2)$ since $A$ and $\mathrm{SL}(2)$ commute and intersect along $K \cong \mu_{2}$. Finally, the scheme-theoretic intersection of $M$ and $T^{\prime}$ is trivial, so the map $M \times T^{\prime} \rightarrow L$ (induced by the inclusions) is an isomorphism.

We now apply Lemma 3.2. Since $H \subseteq L$, the $H$-bundles $E_{1}$ and $E_{2}$ both come from $L$-bundles. Let $D$ (respectively, $D^{\prime}$ ) denote the maximal torus of SL(2) (respectively, GL(2)). Using the form of $\tilde{r}$ in the lemma, we may assume that $E_{1}$ and $E_{2}$ come from $D \times T$-bundles (respectively, $D^{\prime} \times T^{\prime}-$ 


\section{PRINCIPAL BUNDLES ON SINGULAR CURVES}

bundles), differing by a $\mathbb{G}_{m}$-bundle via $\mathbb{G}_{m} \rightarrow D$ (respectively, $\mathbb{G}_{m} \rightarrow D^{\prime}$ ) corresponding to the (standard) coroot of SL(2).

We may clearly ignore the $T$ - and $T^{\prime}$-factors and need to show that the corresponding $\operatorname{SL}(2)$ and GL(2) components, respectively, of $E_{1}$ and $E_{2}$ are isomorphic on $U_{S}$ after Zariski localization in $S$. The Čech cocycles representing $E_{1}$ and $E_{2}$ in the GL(2) case have ratios in $\operatorname{SL}(2)$ and therefore have isomorphic determinant line bundles when considered as rank two vector bundles.

Therefore, in the case of a simply connected $G$, Theorem 1.5 follows from the following assertion: Let $f: X \rightarrow S$ be a proper, flat and finitely presented curve over $S$ and $D \subset X$ a relatively ample effective Cartier divisor which is flat over $S$. Suppose that $E_{1}$ and $E_{2}$ are principal $G$-bundles on $X$ with the same determinant, where (case 1) $G=\mathrm{SL}(2)$ or (case 2) $G=\mathrm{GL}(2)$.

Proposition 3.3. The bundles $E_{1}$ and $E_{2}$ are isomorphic as $G$-bundles on $U$ after a surjective étale base change of $S$. Furthermore, if $S$ is the spectrum of a separably closed field, $E_{1}$ and $E_{2}$ are in the same connected component of the moduli stack of $G$-bundles on $X$.

Proof. We will show that in an étale neighborhood of each $s \in S$, we have, for sufficiently large $n$, exact sequences $(i=1,2)$

$$
0 \rightarrow \mathcal{O} \rightarrow E_{i}(n D) \rightarrow Q_{i} \rightarrow 0 .
$$

Because of our assumptions, $Q_{1}$ and $Q_{2}$ are isomorphic and are trivial on $U$ in case (1). Since $\mathcal{O}(D)$ is relatively ample, the exact sequence (3.2) splits over $U$, with $Q_{i}=\operatorname{det} E_{i}$. The desired conclusions follow: for the deformation, we use the Ext-space $\operatorname{Ext}^{1}\left(Q_{i}, \mathcal{O}\right)$.

We may assume that the residue field of $S$ at $s$ is infinite (in fact, separably closed). We produce such exact sequences by finding nowhere-vanishing global sections $\alpha_{i} \in H^{0}\left(X_{s}, E_{i}(n D)\right)$, where $H^{1}\left(X_{s}, E_{i}(n D)\right)=0$. We choose $n$ sufficiently large so that $H^{1}\left(X_{s}, E_{i}(n D)\right)=0$ and the $E_{i}(n D)$ are globally generated on $X_{s}$ for $i=1,2$. Then the usual Serre argument works: The subset of $H^{0}\left(X_{s}, E_{i}(n D)\right)$ formed by sections that vanish at a point $q$ has codimension $\mathrm{rk} E=2$. Taking the union over all $q \in X_{s}$, the set of "bad sections" lies on a codimension one subvariety of the vector space $H^{0}\left(X_{s}, E_{i}(n D)\right)$.

Remark 3.4. As we explain below, Zariski localization is sufficient in Theorem 1.4 for $G=\operatorname{SL}(n)$ and in Proposition 3.3 in each of the following two cases: (a) the residue fields of $S$ are infinite, and (b) $X \rightarrow S$ is smooth in a neighborhood of $D$.

In case (a), the modification of Proposition 3.3 follows by the same method, and for Theorem 1.4, the method of [Ati57, Theorem 2] and [BL94, Lemma 3.5] applies without changes. Here, we use the fact that the complement of any hypersurface in $\mathbb{A}_{k}^{m}$ has $k$-rational points when $k$ is infinite, so that dimension-counting arguments apply.

In case (b), fix a point $s \in S$. To get the modification of Theorem 1.4, we find everywherelinearly independent sections $\alpha_{1}$ and $\alpha_{2}$ of $E$ on $X_{s}-D_{s}$ by [Ser58, Théorème 1]. Here, $E$ is a vector bundle on $X$ with trivialized determinant. A linear combination $\alpha_{1}+f \alpha_{2}$ (for $f$ a function on $U_{s}$ ) can be found with sufficiently high orders of poles at all points of $D_{s}$, and we obtain a subbundle $\mathcal{O} \subseteq E_{s}\left(n D_{s}\right)$ with $n$ sufficiently large. This can be deformed to a Zariski neighborhood of $s$.

In Proposition 3.3, write (again using [Ser58, Théorème 1]) $E_{i}$ restricted to $U_{s}=X_{s}-D_{s}$ as $\mathcal{O} \alpha_{1}^{(i)} \oplus \mathcal{L}_{i}$, where the $\mathcal{L}_{i}$ are line bundles on $U_{s}$ and the $\alpha_{1}^{(i)}$ are nowhere-vanishing sections. Let $\alpha_{2}^{(i)}$ be a section of $\mathcal{L}_{i}$ on $X_{s}-D_{s}$, for $i=1$, 2. Sections of $\left(E_{i}\right)_{s}$ of the form $\alpha_{1}^{(i)}+f^{(i)} \alpha_{2}^{(i)}$ can be found with sufficiently high orders of poles at all points of $D_{s}$, and these result in subbundles 


\section{P. BELKAle AND N. FAKHRUdDin}

$\mathcal{O} \subseteq\left(E_{i}\right)_{s}\left(n D_{s}\right)$ on $X_{s}$ for $n$ sufficiently large; these can be deformed to a Zariski neighborhood of $s$.

\subsection{Refinements of Theorems 1.1 and 1.5}

In Theorem 1.5, we can replace condition $(\mathrm{C})$ by $\left(\mathrm{C}^{\prime}\right)$ : the $\mathrm{Pic}^{0}$ of the geometric fibers are semiabelian, and draw the weaker conclusion that after a faithfully flat base change $S^{\prime} \rightarrow S$ with $S^{\prime}$ locally of finite presentation over $S$, the bundle $E$ is trivial on $U_{S^{\prime}}$, where $U=X \backslash D$ (see Remark 3.1). This generalizes the flat base change part of [DS95, Theorem 3] and also shows that Theorem 1.1 holds for semi-normal curves without any condition on $\left|\pi_{1}(G)\right|$.

Note that the $\mathrm{Pic}^{0}$ of a projective curve being semi-abelian is equivalent to the curve having geometrically semi-normal singularities [BLR90, Chapter 9.2]; in particular, as is well known, this condition holds for semistable curves.

Now, let $C$ be a (generically reduced) affine curve over an algebraically closed field $k$ of characteristic $p>0$ with a compactification $\bar{C}$ (which is smooth at the boundary) and such that $\operatorname{Pic}^{0}(\bar{C})$ is not semi-abelian. We will show that if $G$ is a semisimple group such that $p$ divides $\left|\pi_{1}(G)\right|$, then there exist non-trivial $G$-bundles on $C$.

Let $\widetilde{G}$ be the simply connected cover of $G$, and let $\widetilde{H}$ be the inverse image in $\widetilde{G}$ of a maximal torus $H$ of $G$. Then $K=\operatorname{ker}(\widetilde{H} \rightarrow H)=\operatorname{ker}(\widetilde{G} \rightarrow G)$ is a finite multiplicative group scheme of order divisible by $p$. The tori $H$ and $\widetilde{H}$ are of the same dimension, say $r$, so $H^{1}(\bar{C}, H)$ and $H^{1}(\bar{C}, \widetilde{H})$ are both isomorphic to $\operatorname{Pic}(\bar{C})^{r}$. Since we have assumed that $p$ divides $\left|\pi_{1}(G)\right|$ and $\operatorname{Pic}^{0}(\bar{C})$ is not semi-abelian, so contains $\mathbb{G}_{a}$ as a subgroup, the map

$$
H^{1}(\bar{C}, \widetilde{H}) \rightarrow H^{1}(\bar{C}, H)
$$

is not surjective and in fact has an infinitely generated cokernel (since this holds for the multiplication by $p$ map on $\left.\operatorname{Pic}^{0}(\bar{C})\right)$. Since $\bar{C}$ is smooth at the boundary points, the surjective map $\operatorname{Pic}(\bar{C}) \rightarrow \operatorname{Pic}(C)$ has a finitely generated kernel. It follows that the image of the second map in the exact sequence

$$
H^{1}(C, \tilde{H}) \rightarrow H^{1}(C, H) \rightarrow H_{\mathrm{fl}}^{2}(C, K)
$$

is not finitely generated. If $E_{H}$ is any $H$-bundle on $C$ which does not lift to an $\widetilde{H}$-bundle, it follows that the induced $G$-bundle $E$ has a non-zero class in $H_{\mathrm{fl}}^{2}(C, K)$; in particular, it is not trivial.

\section{Proof of Theorem 1.6}

We will in fact prove the following (stronger) statement.

THEOREM 4.1. Let $X$ be an algebraic variety (of arbitrary dimension) over an algebraically closed field and $E$ a principal $G$-bundle on $X$, where $G$ is a connected reductive group. Let $\pi: \widetilde{X} \rightarrow X$ be the normalization. Let $R \subset X$ be a reduced subscheme such that $\pi$ is an isomorphism over $U=X \backslash R$. Assume that

(a) $\tilde{X}$ is smooth,

(b) $E$ is generically trivial,

(c) $E$ restricted to $R$ is Zariski-locally trivial (for example, if $\operatorname{dim} R \leqslant 1$ (by Theorem 1.2)).

Then, E is Zariski-locally trivial. 


\section{PRINCIPAL BUNDLES ON SINGULAR CURVES}

By work on the Grothendieck-Serre conjecture [PSV15], one knows that since $\widetilde{E}$, the pull-back of $E$ to $\widetilde{X}$, is generically trivial (by assumption (b)), it is locally trivial in the Zariski topology. In fact, given a finite subset $Z \subset \tilde{X}$, there exists a Zariski-open subset $V \subset \widetilde{X}$ containing $Z$ over which $\widetilde{E}$ is trivial. Let $x \in X$ and $Z=\pi^{-1}(x)$, a finite set. We need to produce an open subset of $X$ containing $x$ over which $E$ has a $B$-structure, where $B$ is a Borel subgroup of $G$. Clearly, we may assume $x \in R$.

Let $V \subset \tilde{X}$ be an open set containing $Z$ over which $\widetilde{E}$ is trivial. Replacing $X$ by an open subset containing $x$ in $X-\pi(\widetilde{X} \backslash V)$, we can assume that $\widetilde{E}$ is trivial on $\widetilde{X}$ and that $X$ is affine.

Let $X_{m}$ be the $m$ th infinitesimal neighborhood of $R$ and $\tilde{X}_{m}=\pi^{-1} X_{m}$. We can now follow the same method of proof as for Theorem 1.1:

- Any section of $\widetilde{E} / B$ on $\widetilde{X}_{m}$ extends to any affine Zariski neighborhood of $Z$ in $\tilde{X}$.

- There exists a section of $E / B$ on any infinitesimal neighborhood $X_{m}$. This is because, by assumption, there is a section of $E / B$ over $R$ (after Zariski localization) that be extended to infinitesimal neighborhoods of $R$. This section can be pulled up to $\widetilde{X}_{m}$.

- For $m$ sufficiently large, a section $s$ of $\tilde{E} / B$ over $\tilde{X}$ descends to a section of $E / B$ over $X$ if the restriction of $s$ to $\widetilde{X}_{m}$ is a pull-back of a section of $E / B$ over $X_{m}$ (the same proof as for Lemma 2.5).

\subsection{An example}

We give examples of normal surfaces $X$ over any algebraically closed field $k$ on which there exist $\operatorname{PGL}(m)$-bundles which are generically trivial but not Zariski-locally trivial.

We begin with some preliminary remarks. First, to prove the existence of $\operatorname{PGL}(m)$-bundles as above (with $m$ not specified), it suffices to construct elements of the Brauer group $\operatorname{Br}(X)$ which are generically trivial but not locally trivial. (That such examples should not be difficult to construct has been suggested by Grothendieck [Gro68, End of Remarques $1.11 \mathrm{c}$ )], but we do not know a reference where this has been made explicit.) Furthermore, by a theorem of Gabber [Gab81], [Hoo82, Corollary 9], for any normal quasi-projective $\operatorname{surface}^{3} X$, the Brauer group is equal to the torsion in $H_{\text {et }}^{2}\left(X, \mathbb{G}_{m}\right)$, so if the characteristic $p$ of $k$ does not divide $n$, the Kummer sequence shows that it suffices to find elements of $H_{\mathrm{et}}^{2}\left(X, \mu_{n}\right)$ which are generically trivial but not locally trivial. We give an example where such elements exist for any such $n>1$.

Let $E \subset \mathbb{P}_{k}^{2}$ be an elliptic curve, and let $p_{1}, p_{2}, \ldots, p_{10}$ be distinct points on $E$. Let $X^{\prime}$ be the blow-up of $\mathbb{P}_{k}^{2}$ at these points, and let $E^{\prime}$ be the strict transform of $E$. We have $\left(E^{\prime}\right)^{2}=-1$, and if there exist an ample line bundle $L$ on $X^{\prime}$ and a positive integer $a$ such that $\left.\left.L\right|_{E^{\prime}} \cong \mathcal{O}\left(-a E^{\prime}\right)\right|_{E^{\prime}}$, then it is well known (and easy to see) that there exists a morphism $\pi: X^{\prime} \rightarrow X$, where $X$ is a normal projective surface, which contracts $E^{\prime}$ and is an isomorphism onto its image when restricted to $X^{\prime} \backslash E^{\prime}$. To ensure this, it suffices, for example, to have positive integers $a^{\prime}$ and $b^{\prime}$ such that $\left.\mathcal{O}_{X^{\prime}}\left(a^{\prime} H\right)\right|_{E^{\prime}}=\mathcal{O}_{E^{\prime}}\left(b\left(p_{1}+p_{2}+\cdots+p_{10}\right)\right)$ in $\operatorname{Pic}(E)$, where $H$ is the pull-back of the class of a line in $\mathbb{P}^{2}$.

We now use the Leray spectral sequence for the map $\pi$ and cohomology with $\mu_{n}$ coefficients. By proper base change, $R^{1} \pi_{*} \mu_{n}$ is a skyscraper sheaf with stalk $(\mathbb{Z} / n)^{2}$ supported on the singular point $p=\pi\left(E^{\prime}\right)$ of $X$. Since $X^{\prime}$ is simply connected, $H_{\mathrm{et}}^{1}\left(X, \mu_{n}\right)=0$, so the differential $d_{2}$ of the spectral sequence gives an embedding of $H^{0}\left(X, R^{1} \pi_{*} \mu_{n}\right)$ into $H_{\mathrm{et}}^{2}\left(X, \mu_{n}\right)$; denote this subgroup

${ }^{3}$ In fact, Gabber has proved this for any quasi-projective variety (unpublished; see [dJo05]) and Schröer has proved this for any geometrically normal (separated) surface. 


\section{P. Belkale And N. FAKhruddin}

by $A$. It is clear that $A$ maps to 0 in $H^{2}\left(X \backslash\{p\}, \mu_{n}\right)$ as well as in $H_{\text {et }}^{2}\left(X^{\prime}, \mu_{n}\right)$. However, we shall show that for all but finitely many $n$, it injects into $H_{\mathrm{et}}^{2}\left(U, \mu_{n}\right)$, where $U$ is any Zariski-open neighborhood of $p$.

Let $\alpha$ be any element of $A$. If $\alpha$ dies in $U$, the Gysin sequence shows that $\alpha$ must be the cohomology class of a divisor $D$ supported in $X \backslash U$. Let $U^{\prime}=\pi^{-1}(U)$; we may view $D$ as a divisor on $X^{\prime}$ since $X \backslash U$ is identified naturally with $X^{\prime} \backslash U^{\prime}$. Since $X^{\prime}$ is a smooth projective rational surface, $H^{2}\left(X^{\prime}, \mathbb{Z} / n\right)=\operatorname{Pic}\left(X^{\prime}\right) / n \operatorname{Pic}\left(X^{\prime}\right)$, so it follows that $D$ must be divisible by $n$ in $\operatorname{Pic}\left(X^{\prime}\right)$. The map $\pi^{*}: \operatorname{Pic}(X) \rightarrow \operatorname{Pic}\left(X^{\prime}\right)$ is an injection, and the cokernel is a finitely generated abelian group. If $n$ is coprime to the order of the torsion part of the cokernel, then $D$ must be divisible by $n$ in $\operatorname{Pic}(X)$, so $\alpha$ must be 0 .

By choosing the $p_{i}$ suitably, one can arrange that the cokernel is torsion free. For example, if $k$ is not $\overline{\mathbb{F}}_{p}$, one can choose $p_{1}, p_{2}, \ldots, p_{9}$ arbitrarily such that $\left.H\right|_{E}, p_{1}, \ldots, p_{9}$ are independent in $\operatorname{Pic}(E)$ and then choose $p_{10}$ to satisfy the equation $\left.10 H\right|_{E}=3\left(p_{1}+p_{2}+\cdots+p_{10}\right)$ in $\operatorname{Pic}(E)$. In general, one has $\operatorname{Pic}(X)=\operatorname{Ker}\left(\operatorname{Pic}\left(X^{\prime}\right) \rightarrow \operatorname{Pic}\left(E^{\prime}\right)\right)$; with the above choice, one then sees that $\operatorname{Pic}(X)$ is of rank one and the cokernel is torsion free.

Remark 4.2. Given an $\alpha$ as above of order $n$, we do not know what is the smallest integer $m$ such that $\alpha$ comes from a $\operatorname{PGL}(m)$ torsor on $X$.

\section{Applications}

Proposition 5.1. Let $\bar{C}$ be a projective curve over an algebraically closed field $k$ and $G$ a semisimple simply connected group. The moduli stack $\operatorname{Bun}_{G}(\bar{C})$ of principal $G$-bundles on $\bar{C}$ is connected.

Note. The moduli stack $\operatorname{Bun}_{G}(\bar{C})$ is well known to be smooth; see, for example, [Wan11]!1 ${ }^{\text {hote }}$

Proof. The proof of [DS95, Proposition 5] generalizes easily (using Theorem 1.2) with one small change in detail.

Let $B$ be a Borel subgroup of $G$ and $H \subset B$ a maximal torus. Let Bun $_{G}$, Bun $_{H}$ and Bun Bu $_{B}$ de note the corresponding stacks of bundles on $\bar{C}$. The pointed set $\pi_{0}\left(\operatorname{Bun}_{B}\right)$ surjects onto $\pi_{0}\left(\operatorname{Bun}_{G}\right)$ (by Theorem 1.2), and $\pi_{0}\left(\operatorname{Bun}_{H}\right)$ is in bijection with $\pi_{0}\left(\operatorname{Bun}_{B}\right)$ (as in [DS95]). Therefore, it suffices to connect the image of an $H$-bundle in $\operatorname{Bun}_{G}$ to the trivial $G$-bundle. To do this, we need to show (since coroots generate cocharacters) that if we twist an $H$-bundle by a cocharacter, the image of the $H$-bundle (in $\operatorname{Bun}_{G}$ ) stays in the same connected component. This follows from Proposition 3.3 (see Section 3.2.2).

Assume for the rest of this section that $\bar{C}$ is a reduced projective curve over $k=\mathbb{C}$. Let $p_{1}, \ldots, p_{n}$ be smooth points of $\bar{C}$ such that $C=\bar{C}-\left\{p_{1}, \ldots, p_{n}\right\}$ is affine. Choose local uniformizing parameters at the points $p_{i}$.

Consider the $k$-groups $L_{G}^{C}, L_{G}$ and $L_{G}^{+}$which assign to a $k$-algebra $R$ the groups $G(\Gamma(C, \mathcal{O})$ $\otimes R), G(R((z)))$ and $G(R[[z]])$, respectively. Then $L_{G}$ is an ind-scheme, ${ }^{4}$ and $L_{G}^{+}$is an affine scheme. There is an embedding $L_{G}^{C} \rightarrow\left(L_{G}\right)^{n}$, and the scheme $L_{G}^{C}$ inherits an ind-scheme structure from $\left(L_{G}\right)^{n}$.

\footnotetext{
${ }^{4} \mathrm{An}$ ind-scheme in this paper is a $k$-space (that is, a set-valued functor on the category of commutative $k$-algebras) of the form $\lim Y_{n}$, indexed by the natural numbers, such that each $Y_{n}$ is a scheme over $k$ and all the maps $Y_{n} \rightarrow Y_{n+1}$ are closed embeddings.
} 


\section{PRINCIPAL BUNDLES ON SINGULAR CURVES}

Let $\mathcal{Q}_{G}=L_{G} / L_{G}^{+}$be the affine Grassmannian. As a corollary of Theorem 1.5, we obtain the following generalization of the uniformization theorem [BL94, BL95, LS97, DS95] for singular curves.

Proposition 5.2. Suppose that $G$ is semisimple (possibly not simply connected). There is an isomorphism of stacks $L_{G}^{C} \backslash\left(\mathcal{Q}_{G}\right)^{n} \stackrel{\sim}{\rightarrow} \operatorname{Bun}_{G}(\bar{C})$.

The morphism is constructed using [BL95] (note that $p_{1}, \ldots, p_{n}$ are smooth points of $\bar{C}$ ). That it is an isomorphism follows from Theorem 1.4.

The following is a generalization of a result of Laszlo and Sorger [LS97, Proposition 5.1].

Proposition 5.3. Assume that $G$ is simple and simply connected. The $k$-group $L_{G}^{C}$ is integral as an ind-scheme, that is, can be written as a limit of an increasing sequence of integral schemes.

Proof. Following the proof of [LS97] (and Theorem 1.4), we see that $\left(\mathcal{Q}_{G}\right)^{n} \rightarrow \operatorname{Bun}_{G}(\bar{C})$ is locally (in the étale topology) a fiber bundle with fiber $L_{G}^{C}$. This shows that $L_{G}^{C}$ is reduced. Connected ind-groups are irreducible by a theorem of Shafarevich [Sha82]; therefore, it suffices (exactly as in [LS97]) to show that $L_{G}^{C}$ is connected.

We follow the proof in [LS97] of the connectedness of $L_{G}^{C}$ when $C$ is smooth (attributed there to Drinfeld). Let $C^{\prime}=C-\{p\}$, where $p$ is a smooth point. Then, using Theorem 1.4, we see that

$$
L_{G}^{C^{\prime}} / L_{G}^{C} \stackrel{\sim}{\rightarrow} \mathcal{Q}_{G},
$$

exactly as in [LS97], and one obtains that the (sets of) connected components of $L_{G}^{C}$ and $L_{G}^{C^{\prime}}$ are in bijection. Therefore, removing any finite number of smooth points of $C$ does not change the number of connected components of $L_{G}^{C}$.

Let $g \in L_{G}^{C}(k)$. Let $Z$ be the set of singularities of $C$ and $K_{Z}$ the semilocal ring at $Z$. These are rational functions on $C$ which are regular at points of $Z$. Now, $g$ gives rise to a point in $G\left(K_{T}\right)$. It is known by the works [IM65, Ste73] (see also the recent article [SSV12]) that $G\left(K_{Z}\right)$ is generated by $U^{+}\left(K_{Z}\right)$ and $U^{-}\left(K_{Z}\right)$, where $\delta_{ \pm}: \mathbb{A}_{k}^{m} \stackrel{\sim}{\rightarrow} U^{ \pm}$are the unipotent radicals of a Borel $B^{+}$and an opposite $B^{-}$. Therefore, we have maps

$$
u_{i}: C^{\prime \prime} \rightarrow \mathbb{A}_{k}^{m}, \quad i=1, \ldots, s,
$$

such that $g=\delta_{ \pm} u_{1} \cdots \delta_{ \pm} u_{s}$ and $C^{\prime \prime}=C-\left\{q_{1}, \ldots, q_{m}\right\}$, where the $q_{i}$ are smooth points of $C$. Since the sets of connected components of $L_{G}^{C}$ and $L_{G}^{C^{\prime \prime}}$ are in bijection, we just need to connect $g$ and 1 in $L_{G}^{C^{\prime \prime}}$. For this, it suffices to consider the map $\mathbb{A}_{k}^{1} \rightarrow L_{G}^{C^{\prime \prime}}$ given by

$$
(t, x) \mapsto \delta_{ \pm}\left(t u_{1}(x)\right) \cdots \delta_{ \pm}\left(t u_{s}(x)\right) .
$$

\section{Conformal blocks and generalized theta functions}

Let $\overline{\mathcal{M}}_{g, n}$ be the moduli stack parameterizing stable $n$-pointed curves $(C ; \vec{p})=\left(C, p_{1}, \ldots, p_{n}\right)$ of genus $g$. In this section, $k=\mathbb{C}$.

\subsection{Conformal blocks}

Let $G$ be a simple, connected, simply connected complex algebraic group with Lie algebra $\mathfrak{g}$, let $B$ be a Borel subgroup of $G$ and $H \subset B$ a maximal torus. For an integer (called the level) $\ell \geqslant 0$, let $P_{\ell}(\mathfrak{g})$ denote the set of dominant integral weights $\lambda$ with $(\lambda, \theta) \leqslant \ell$, where $\theta$ is the highest root and $($,$) is the Killing form, normalized so that (\theta, \theta)=2$. Let $\hat{\mathfrak{g}}$ be the Kac-Moody central extension of $\mathfrak{g}$ (see, for example, [Sor96]). For a dominant integral weight $\lambda$ in $P_{\ell}(\mathfrak{g})$, let $\mathcal{H}_{\lambda, \ell}$ 


\section{P. BELKAle AND N. FAKhruddin}

denote the corresponding irreducible representation of $\hat{\mathfrak{g}}$. Note that $V_{\lambda} \subseteq \mathcal{H}_{\lambda, \ell}$, where $V_{\lambda}$ is the corresponding irreducible representation of $\mathfrak{g}$.

Consider an $n$-tuple $\vec{\lambda}=\left(\lambda_{1}, \ldots, \lambda_{n}\right)$ of elements in $P_{\ell}(\mathfrak{g})$. Corresponding to this data, there are vector bundles of conformal blocks $\mathbb{V}=\mathbb{V}_{\mathfrak{g}, \vec{\lambda}, \ell}$ on the moduli stacks $\overline{\mathcal{M}}_{g, n}$ of stable $n$-pointed curves of arbitrary genus $g$ [TUY89, Fak12] (we will suppress the genus $g$ in the notation of conformal block bundles). The fiber of $\mathbb{V}$ over $\left(C, p_{1}, \ldots, p_{n}\right) \in \overline{\mathcal{M}}_{g, n}$ is a finite-dimensional quotient (after choosing local coordinates at $p_{i}$ ) of the form

$$
\otimes_{i=1}^{n} \mathcal{H}_{\lambda_{i}, \ell} / \mathfrak{g} \otimes \Gamma\left(C-\left\{p_{1}, \ldots, p_{n}\right), \mathcal{O}\right) \otimes_{i=1}^{n} \mathcal{H}_{\lambda_{i}, \ell}
$$

where $\mathfrak{g} \otimes \Gamma\left(C-\left\{p_{1}, \ldots, p_{n}\right), \mathcal{O}\right)$ acts on $\otimes_{i=1}^{n} \mathcal{H}_{\lambda_{i}, \ell}$ via an embedding into $\oplus_{i=1}^{n} \hat{\mathfrak{g}}$ (see [TUY89]).

\subsection{Moduli stacks of parabolic bundles}

Definition 6.1. Consider an $n$-pointed reduced projective curve $(C ; \vec{p})=\left(C, p_{1}, \ldots, p_{n}\right)$, where $p_{1}, \ldots, p_{n}$ are distinct smooth points of $C$. Let $\operatorname{Parbun}_{G}(C ; \vec{p})=\operatorname{Parbun}_{G}\left(C, p_{1}, \ldots, p_{n}\right)$ denote the moduli stack parameterizing tuples $\left(E, \tau_{1}, \ldots, \tau_{n}\right)$, where $E$ is a principal $G$-bundle on $C$ and $\tau_{i} \in E_{p_{i}} / B$ for $i=1, \ldots, n$.

Performing this construction in families of stable $n$-pointed curves, we obtain stacks $\operatorname{Parbun}_{G, g, n}$ (in the faithfully flat and of finite presentation (fppf) topology) with morphisms

$$
\pi: \operatorname{Parbun}_{G, g, n} \rightarrow \overline{\mathcal{M}}_{g, n}
$$

such that $\pi^{-1}(C ; \vec{p})=\operatorname{Parbun}_{G}(C ; \vec{p})$. Since $\operatorname{Parbun}_{G, g, n} \rightarrow \operatorname{Parbun}_{G, g, 0}=\operatorname{Bun}_{G, g}$ is a representable morphism with smooth fibers and $\operatorname{Bun}_{G, g}$ is an Artin stack that is smooth over $\overline{\mathcal{M}}_{g, n}$ (see, for example, [Wan11]), it follows that $\operatorname{Parbun}_{G, g, n}$ is also an Artin stack that is smooth over $\overline{\mathcal{M}}_{g, n}$.

Our aim in this section is to construct a line bundle $\mathcal{L}=\mathcal{L}_{\mathfrak{g}, \vec{\lambda}, \ell}$ on $\operatorname{Parbun}_{G, g, n}$ and to construct an isomorphism

$$
\pi_{*} \mathcal{L} \stackrel{\sim}{\rightarrow} \mathbb{V}_{\mathfrak{g}, \lambda, \ell}^{*}
$$

In fact, we will construct such line bundles and isomorphisms (compatibly with pull-backs) for arbitrary families of stable $n$-pointed curves.

Over $\mathcal{M}_{g, 1}$ and for $\lambda_{1}=0$, such line bundles and isomorphisms were given by Laszlo [Las98, Section 5], building upon the case of a single curve considered by many authors. Sorger's construction [Sor99] of the line bundle via the theory of conformal blocks plays an important role in Laszlo's construction. Our approach to (6.1) generalizes Laszlo's work [Las98].

Remark 6.2. The functor $\pi_{*}$ is constructed using [LM00, Section (12.5)] and [Ols07].

\subsection{Construction of the line bundle}

Fix a family of stable $n$-pointed curves $C \rightarrow S$, with the pointed sections denoted by $\sigma_{i}: S \rightarrow C$. Localizing in the étale topology on $S$, we will make a further choice of disjoint sections $\tau_{1}, \ldots, \tau_{m}$ disjoint from $\sigma_{1}, \ldots, \sigma_{n}$ such that

- the curve $C^{\prime}=C-\cup_{i=1}^{m} \tau_{i}(S)-\cup_{j=1}^{n} \sigma_{j}$ is affine over $S$,

- the pointed curves in the family have no automorphisms.

We will construct a line bundle $\mathcal{L}$ on $\operatorname{Parbun}_{S}=\left(\operatorname{Parbun}_{G, g, n}\right)_{S}$, the stack of parabolic bundles over $C \rightarrow S$, and show that the line bundle is independent (with canonical isomorphisms) 


\section{PRINCIPAL BUNDLES ON SINGULAR CURVES}

of the choice of the extra sections $\tau_{1}, \ldots, \tau_{m}$, allowing us (by descent) to define a line bundle without assuming the existence of such sections $\tau_{1}, \ldots, \tau_{m}$. Set $\sigma_{n+a}=\tau_{a}$ for $a=1, \ldots, m$.

First, assume that $S$ is the spectrum of a ring $R$. For an $R$-algebra $A$, let $C_{A}=C \times{ }_{R} \operatorname{Spec}(A)$ and $C_{A}^{\prime}=C^{\prime} \times{ }_{R} \operatorname{Spec}(A)$. We have an $R$-group $L_{C^{\prime}}^{G}$ (see [BL94, Dri06]) which assigns to every $R$-algebra $A$ the group

$$
L_{C^{\prime}}^{G}(A)=G\left(\Gamma\left(C_{A}^{\prime}, \mathcal{O}\right)\right)=\operatorname{Mor}_{k}\left(C_{A}^{\prime}, G\right) .
$$

We also have ind-schemes $\mathcal{I} \subseteq \mathcal{L} \mathcal{G}$ over $S$ whose $A$-points are defined as follows. Let $\sigma_{1}^{A}, \ldots$, $\sigma_{n+m}^{A}$ be sections of $C_{A} \rightarrow \operatorname{Spec}(A)$ obtained by base change. Let $\widehat{C}_{A}$ be the completion of $C_{A} \rightarrow$ $\operatorname{Spec}(A)$ along the union of these sections and $\widehat{C}_{A}^{\prime}$ the complement of the union of the induced sections of $\widehat{C}_{A} \rightarrow \operatorname{Spec}(A)$.

- Let $\mathcal{L} \mathcal{G}(A)=G\left(\Gamma\left(\widehat{C}_{A}^{\prime}, \mathcal{O}\right)\right)$.

- The Iwahori group $\mathcal{I}(A)$ is defined to be subgroup of elements of $G\left(\Gamma\left(\widehat{C}_{A}, \mathcal{O}\right)\right)$ which map to points of $B(A)^{n+m}$ under the natural map $G\left(\Gamma\left(\widehat{C}_{A}, \mathcal{O}\right)\right) \rightarrow G(A)^{n}$.

If local coordinates $z_{i}$ are chosen along $\sigma_{i}$, we may identify $\mathcal{L G}(A)$ and $\mathcal{I}(A)$ with $\prod_{i=1}^{n+m} G\left(A\left(\left(z_{i}\right)\right)\right)$ and $\prod_{i=1}^{n+m} I(A)$, respectively, where $I(A)$ is the inverse image of $B(A)$ under the morphism $G(A[[z]]) \rightarrow G$.

Proposition 6.3. The $R$-group $L_{C^{\prime}}^{G}$ is (relatively) ind-affine and formally smooth with connected integral geometric fibers over $S=\operatorname{Spec}(R)$.

Proof. We first claim that the coordinate ring of $C^{\prime}$ is a direct limit of (Zariski-)locally free $R$-modules

with $M_{i}$ a local direct summand of $M_{i+1}$.

$$
\underset{\lim _{i+1}}{\longrightarrow} M_{i}, \quad i=1,2, \ldots
$$

It suffices to prove this claim in the universal case where we have a flat family of pointed curves over an integral variety (since the family consists of rigid pointed curves). In this case, the coordinate ring of $C^{\prime}$ can be filtered by order of pole along the total boundary divisor (which is ample), and then standard cohomology and base change arguments give the desired direct limit (we could instead have used a theorem of Lazard that flat modules over any ring are filtered limits of finitely generated free modules [Laz64]).

The argument for the case of a field (which embeds $G$ into a general linear group) generalizes to give the ind-affine property: The matrix coefficients of the $R$-points of $L_{C^{\prime}}^{G}$ in the embedding in a general linear group are filtered by the filtration of the coordinate ring. The equations defining $G$ in the general linear group now give the desired ind-affine structure.

For formal smoothness, we need to show that $L_{C^{\prime}}^{G}(A / I) \rightarrow L_{C^{\prime}}^{G}(A)$ is surjective whenever $A$ is an $R$-algebra with a nilpotent ideal $I$. Pick $\phi: C_{A / I}^{\prime} \rightarrow G$ in $L_{C^{\prime}}^{G}(A / I)$. Now, $C_{A / I}^{\prime} \rightarrow C^{\prime}{ }_{A}$ is a morphism $\operatorname{Spec}\left(B^{\prime}\right) \rightarrow \operatorname{Spec}(B)$ with $B^{\prime}=B / J$ with $J \subset B$ nilpotent. Since $G$ is a smooth scheme over the base field, the desired surjection follows.

The assertion on integrality of geometric fibers follows from Proposition 5.3.

\subsection{Central extensions}

Using results of Faltings ([BL94, Lemma 8.3], also [LS97]), we see that $\mathcal{L G}$ has a projective representation (this construction is coordinate independent) on

$$
\mathcal{H}=R \otimes_{k}\left(\otimes_{i=1}^{n+m} \mathcal{H}_{\lambda_{i}, \ell}\right)
$$




\section{P. BELKAle AND N. FAKhruddin}

lifting a natural representation of affine Kac-Moody algebras in the following sense. Suppose that $A$ is an $R$-algebra, hence a $k$-algebra, and $\gamma \in \mathcal{L G}(A)$. Then locally (in the Zariski topology) on $\operatorname{Spec}(A)$, there is an automorphism $u_{\gamma}$ of $\mathcal{H}_{A}=\mathcal{H} \otimes_{R} A$, unique up to units $R^{\times}$, such that for all $\alpha$ in the $A$-valued points of the corresponding Kac-Moody Lie algebra (which, if local coordinates are chosen, is $\left.A c \oplus \oplus_{i} \mathfrak{g} \otimes A\left(\left(z_{i}\right)\right)\right)$, the following diagram commutes:

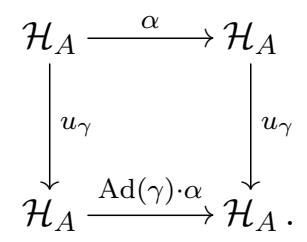

This gives rise to a representation

$$
\mathcal{L} \mathcal{G} \rightarrow \operatorname{PGL}(\mathcal{H})
$$

whose derivative coincides with the natural action of the Lie algebra of $\mathcal{L G}$ on $\mathcal{H}$ (up to scalars).

Let $\widehat{\mathcal{L G}}$ be the corresponding central extension of $\mathcal{L G}$ :

$$
1 \rightarrow \mathbb{G}_{m} \rightarrow \widehat{\mathcal{L G}} \rightarrow \mathcal{L} \mathcal{G} \rightarrow 1
$$

The extension (6.3) splits over $\mathcal{I} \subseteq \mathcal{L} \mathcal{G}$ because the projective ambiguity disappears over $\mathcal{I}$ (the action over the tensor product of highest weight vectors can be normalized; see [Sor00, Lemma 7.3.5]). We require $\mathcal{I}$ to act on the tensor product of highest weight vectors via the map to $B(A)^{n+m}$ using the product of characters $\lambda_{i}: B \rightarrow \mathbb{G}_{m}$. Therefore, $\mathcal{I}$ is a subgroup of $\widehat{\mathcal{L G}}$.

Let $\mathcal{I}_{G}=G(k((z))) / I$ be the Iwahori Grassmannian. It is known ([Mat88], also [KNR94] and [PR08, Proposition 10.1]) that the Picard group of $\mathcal{I}_{G}$ is a direct $\operatorname{sum} \mathbb{Z} \oplus \operatorname{Pic}(G / B)$, where the first factor is generated by line bundles from the affine Grassmannian and the second factor comes from characters of $B$. The $\mathbb{Z}$-component of an element of the Picard group will be referred to as the level of the line bundle.

Remark 6.4. The Lie algebra of $\widehat{\mathcal{L G}}$ is (canonically) isomorphic to the direct sum of affine Lie algebras $\hat{\mathfrak{g}}$ modulo the sum of central elements (considered in families, in a coordinate-free way); see [Las98, Proposition 4.6].

We remark that $\widehat{\mathcal{L G}}$ is itself independent of the choice of the $\lambda_{i}$ (at the same level). The main point is that the Iwahori Grassmannian $\widehat{\mathcal{L G}} / \widehat{\mathcal{I}}=\mathcal{L G} / \mathcal{I}=\mathcal{I}_{G}^{n+m} \times_{k} S$ is independent of the choice of the $\lambda_{i}$. To see this, note that $\widehat{\mathcal{L G}}$ is a Mumford group (with given isomorphisms) for all line bundles (trivialized over the identity coset) on $\mathcal{L} \mathcal{G} / \mathcal{I}=\mathcal{I}_{G}^{n+m} \otimes_{k} S$ with all components at the given level $\ell$ (following [LS97, Section 8.3]), lifting the natural action of $\mathcal{L G}$ on $\mathcal{L G} / \mathcal{I}$.

The following generalizes a result of Sorger [Sor99].

Lemma 6.5. The extension (6.3) splits over $L_{C^{\prime}}^{G} \subseteq \mathcal{L G}$.

Proof. Let $\mathbb{V}=\mathbb{V}_{\mathfrak{g}, \vec{\lambda}, \ell}$ be the sheaf of conformal blocks at level $\ell$ on $S$ associated to the data $\left(\lambda_{1}, \ldots, \lambda_{n}, 0^{m}\right)$. Recall that $\mathbb{V}$ is a quotient of $\mathcal{H}$. By Remark 6.4, changing $\lambda_{i}$ at a fixed level $\ell$ does not change $\widehat{\mathcal{L G}}$; therefore, we may assume $\mathbb{V} \neq 0$ (since this is true if $\lambda_{1}=\cdots=\lambda_{n}=0$ ).

Let

$$
1 \rightarrow \mathbb{G}_{m} \rightarrow \mathrm{GL}(\mathbb{V}) \rightarrow \operatorname{PGL}(\mathbb{V}) \rightarrow 1
$$

be the corresponding central extension. 


\section{PRINCIPAL BUNDLES ON SINGULAR CURVES}

The projective representation of $L_{C^{\prime}}^{G}$ on $\mathcal{H}$ passes to a projective representation on $\mathbb{V}$,

$$
\mathbb{V} \otimes_{R} A=\mathcal{H}_{A} / \operatorname{Lie}\left(L_{C^{\prime}}^{G}\right)(A) \otimes_{A} \mathcal{H}_{A}
$$

which can be seen using

$$
\operatorname{Lie}\left(L_{C^{\prime}}^{G}\right)(A)=\mathfrak{g} \otimes_{k} \Gamma\left(C_{A}^{\prime}, \mathcal{O}\right)
$$

and diagram (6.2) with $\gamma \in L_{C^{\prime}}^{G}(A)$ and $\alpha \in \mathfrak{g} \otimes \Gamma\left(C_{A}^{\prime}, \mathcal{O}\right)=\operatorname{Lie}\left(L_{C^{\prime}}^{G}\right)(A)$.

The derivative of the projective representation of $L_{C^{\prime}}^{G}$ on $\mathbb{V}$ is zero. This is because the map $\mathcal{L G} \rightarrow \operatorname{PGL}(\mathcal{H})$ has derivative which is, up to scalars, given by the natural action of the affine Kac-Moody algebra on $\mathcal{H}$. The induced morphism $L_{C^{\prime}}^{G} \rightarrow \operatorname{PGL}(\mathbb{V})$ is therefore trivial (Proposition 6.12). This allows us to fix the projective ambiguity in the action of $L_{C^{\prime}}^{G}$ on $\mathcal{H}$, so that the corresponding action on $\mathbb{V}$ is actually trivial.

\subsection{Line bundles}

Set $\hat{\mathcal{I}}=\mathcal{I} \times \mathbb{G}_{m}$. The character $\hat{\mathcal{I}}=\mathcal{I} \times \mathbb{G}_{m} \rightarrow \mathbb{G}_{m}$ given by the inverse on $\mathbb{G}_{m}$ and the inverse of product of characters $\lambda_{i}\left(\right.$ and $\left.0^{m}\right)$ on $\mathcal{I}$ produces a line bundle on the quotient stack

$$
\widetilde{\mathcal{Q}}=\widehat{\mathcal{L G}} / \widehat{\mathcal{I}}=\mathcal{L} \mathcal{G} / \mathcal{I}
$$

It is easy to see that $\widetilde{\mathcal{Q}}$ is a fiber bundle over an $(n+m)$-fold product of the affine Grassmannian $\mathcal{Q}_{G} \times{ }_{k} S$ (with fibers $(G / B)^{n+m}$ ). In particular, it is a formally smooth ind-scheme over $S$.

Generalizing Proposition 5.2, we have the following.

Proposition 6.6. The stack quotient $L_{C^{\prime}}^{G} \backslash \widetilde{\mathcal{Q}}$ is the pull-back of the stack $\operatorname{Parbun}_{G, g, n+m}$ to $S$.

The proof again uses [BL95] and Theorem 1.4. In particular, we obtain a line bundle $\mathcal{L}$ on the pull-back of $\operatorname{Parbun}_{G, g, n+m}$ to $S$.

We can also identify $\widetilde{\mathcal{Q}}$ with an $(n+m)$-fold product of the Iwahori Grassmannian, and the line bundle $\mathcal{L}$ pulls back to the line bundle corresponding to $\lambda_{i}$ at level $\ell$ on this Iwahori Grassmannian. This leads to (by work of Kumar [Kum87] and Mathieu [Mat88])

$$
H^{0}(\widetilde{\mathcal{Q}}, \mathcal{L})=\mathcal{H}^{*}
$$

In particular, using Propositions 5.3, 6.6 and 6.11 (and equations (6.4), and (6.5)), we get

$$
H^{0}\left(\left(\operatorname{Parbun}_{G, g, n+m}\right)_{S}, \mathcal{L}\right)=\mathbb{V}^{*} .
$$

Remark 6.7. The line bundles obtained above on $\widetilde{\mathcal{Q}}$ are trivialized along the identity coset. Similarly, the line bundle on $\operatorname{Parbun}_{G, g, n+m}$ is trivialized along the trivial section of $\operatorname{Parbun}_{G, g, n+m}$ over $S$. Such rigidifications fix the line bundle up to canonical isomorphisms.

Adding one more section $\tau$ (after further affine étale localization $S^{\prime}$ of $S$ ) leads to a line bundle $\mathcal{L}^{\prime}$ on $\operatorname{Parbun}_{G, g, n+m+1}$. It it is easy to see (using the propagation of vacua isomorphisms) that the pull-back of $\mathcal{L}^{\prime}$ to $L_{C^{\prime}}^{G} \backslash \widetilde{\mathcal{Q}}$ (pulled up to $S^{\prime}$ ) is canonically identified with $\mathcal{L}$.

\subsection{Proof of Theorem 1.7}

We now use a descent technique from [Fak12] (see the discussion following Proposition 2.1 in [Fak12]) to show the independence of $\mathcal{L}$ from the choice of sections $\tau_{1}, \ldots, \tau_{m}$. Suppose that we are given two sets of the $\tau$, say $\tau_{1}, \ldots, \tau_{m}$ and $\tau_{1}^{\prime}, \ldots, \tau_{m^{\prime}}^{\prime}$. Add a further large collection of sections $\sigma_{1}, \ldots, \sigma_{s}$ to each of these sets, disjoint from the earlier sections (after étale localization 


\section{P. BELKAle AND N. FAKHRUdDin}

in $S$ ). We have isomorphisms of the $\mathcal{L}$ coming from $\tau_{1}, \ldots, \tau_{m}$ with that coming from $\tau_{1}, \ldots, \tau_{m}$, $\sigma_{1}, \ldots, \sigma_{s}$, which is in turn canonically isomorphic to the line bundle coming from $\sigma_{1}, \ldots, \sigma_{s}$, and similarly for $\tau_{1}^{\prime}, \ldots, \tau_{m^{\prime}}^{\prime}$. This gives the patching data (the cocycle condition is easy to check).

Therefore, there exists (by descent) a line bundle $\mathcal{L}$ on the stack $\operatorname{Parbun}_{G, g, n}$. It is now easy to see that the desired isomorphism (6.1) holds. This completes the proof of Theorem 1.7.

It also follows that the line bundle $\mathcal{L}$ can be defined for arbitrary bases $S$. To prove the isomorphism (6.1) over a base, we may localize and assume $S$ to be affine and apply (6.6) when $S$ is of finite type over $k$ (the finite-type requirement comes from Proposition 6.11).

Remark 6.8. We may show that (6.6) holds for arbitrary affine $S$ (not necessarily of finite type over $k$ ) as follows. The implication $(\mathrm{a}) \Rightarrow(\mathrm{b})$ in Proposition 6.11 works for arbitrary affine $S$, and hence there is an injective map (6.6) for arbitrary $S$. For the surjection, we can assume, since the sheaves of covacua $\mathbb{V}^{*}$ are locally free of finite rank (and commute with base change), that there is an $R$-basis of the sheaf of vacua coming from a family over a finitely generated base. Therefore, any particular element of $\mathbb{V}^{*}$ is the pull-back from an affine scheme $S^{\prime}$ of finite type over $k$ via a map $S \rightarrow S^{\prime}$. We can now apply the isomorphism (6.6) for $S^{\prime}$ and the pull-back map $\left(\operatorname{Parbun}_{G, g, n+m}\right)_{S} \rightarrow\left(\operatorname{Parbun}_{G, g, n+m}\right)_{S^{\prime}}$ to conclude that (6.6) is surjective for all schemes $S$ over $k$.

\subsection{Explicit description of the line bundles $\mathcal{L}$ on $\operatorname{Parbun}_{G, g, n}$}

Let $V$ be an irreducible representation of $G$ with Dynkin index $d_{V}$ (see [KNR94]). Consider the line bundle $\mathcal{L}^{\prime}$ on $\operatorname{Parbun}_{G, g, n+m}$, which over a marked curve $(C, \vec{p})$ and point $\left(E, \tau_{1}, \ldots, \tau_{n}\right) \in$ $\operatorname{Parbun}_{G}(C ; \vec{p})$ equals the tensor product of

- the line $\left(\operatorname{det}\left(H^{*}\left(C, E \times_{G} V\right)\right) \otimes \operatorname{det}\left(H^{*}(C, V \otimes \mathcal{O})\right)^{-1}\right)^{\ell}$ (here, for a coherent sheaf $W$ on $C$, the term $\operatorname{det}\left(H^{*}(C, W)\right)$ is the line

$$
\left.\bigwedge^{\text {top }} H^{0}(C, W)^{*} \otimes \bigwedge_{\text {top }} H^{1}(C, W)\right) .
$$

- the lines (for $i=1, \ldots, n$ ) obtained as fibers of $E_{p_{i}} \times_{B} \mathbb{C}_{-\lambda_{i}} \rightarrow E_{p_{i}} / B$ over the given elements $\tau_{i}$.

It is easy to see (using Remark 6.7 and Lemma 7.2) that $\mathcal{L}^{\prime}$ equals the line bundle $\mathcal{L}$ on $\operatorname{Parbun}_{G, g, n+m}$ associated to the data $\vec{\lambda}$ but at level $\ell d_{V}$. Therefore, as a special case, Theorem 1.7 gives the following.

Theorem 6.9. We have $\pi_{*} \mathcal{L}^{\prime}=\mathbb{V}_{\mathfrak{g}, \vec{\lambda}, \ell d_{V}}$.

Similarly to Theorem 1.7, the version for arbitrary families of $n$-pointed curves also holds.

When $G$ is a special linear or symplectic group and $V$ is the standard representation, we have $d_{V}=1$. The standard (vector) representation for Spin groups has $d_{V}=2$, and therefore odd levels are not covered by Theorem 6.9 (but they are covered by Theorem 1.7).

The theory of Pfaffian line bundles [LS97] for spin groups and fixed smooth curves to cover odd levels seems to run into difficulties for singular curves for degree reasons: the degree of the dualizing sheaf of a reducible curve may be odd on irreducible components.

\subsection{Generalities on ind-group actions}

Let $S=\operatorname{Spec} R$, and suppose that $\Gamma$ is an ind-group over $S$ acting on an ind-scheme $\mathcal{Q}$ over $S$. Let $\mathcal{L}$ be a $\Gamma$-linearized line bundle on $\mathcal{Q}$. In this setting, $\operatorname{Lie}(\Gamma)(R)=\operatorname{ker}\left(\Gamma\left(R[\epsilon] / \epsilon^{2}\right) \rightarrow \Gamma(R)\right)$ acts on 


\section{PRINCIPAL BUNDLES ON SINGULAR CURVES}

$H^{0}(\mathcal{Q}, \mathcal{L})$ (note that since $R^{\prime}=R[\epsilon] /\left(\epsilon^{2}\right)$ is a free $R$-algebra, $\left.H^{0}\left(\mathcal{Q}_{R^{\prime}}, \mathcal{L}_{R^{\prime}}\right)=H^{0}(\mathcal{Q}, \mathcal{L}) \otimes_{R} R^{\prime}\right)$. We will make the following assumptions in this section:

- Assume that $\Gamma$ and $\mathcal{Q}$ are inductive limits of schemes of finite type over $S$ and that $S$ is a scheme of finite type over $k=\mathbb{C}$.

- Assume that $\Gamma$ is ind-affine and formally smooth over $S$ with integral geometric fibers,

Definition 6.10. A section $s \in H^{0}(\mathcal{Q}, \mathcal{L})$ is $\Gamma$-invariant if $\Gamma(A)$ acts trivially on the pullback section $s_{A}$ of $H^{0}\left(\mathcal{Q}_{A}, \mathcal{L}_{A}\right)$ for all $R$-algebras $A$. This definition can be reformulated as the vanishing of a suitable section of the pull-back of $\mathcal{L}$ on $\Gamma \times{ }_{S} \mathcal{Q}$ [BL94, Lemma 7.2].

By [BL94, Proposition 7.2], the space of such sections is in bijection with $H^{0}\left(\Gamma \backslash \mathcal{Q}, \mathcal{L}_{0}\right)$, where $\mathcal{L}_{0}$ is the line bundle on the stack $\Gamma \backslash \mathcal{Q}$ obtained via descent from $\mathcal{L}$. Note that in this generality, $\Gamma \backslash \mathcal{Q}$ may not be algebraic.

The following is a generalization of [BL94, Proposition 7.4].

Proposition 6.11. Let $s \in H^{0}(\mathcal{Q}, \mathcal{L})$. The following are equivalent:

(a) The section $s$ is $\Gamma$-invariant.

(b) For every $R$-algebra $A$, the pull-back section $s_{A}$ of $H^{0}\left(\mathcal{Q}_{A}, \mathcal{L}_{A}\right)$ is invariant under the Lie algebra $\operatorname{Lie}(\Gamma)(A)$ (which is the kernel of $\left.\Gamma\left(A[\epsilon] /\left(\epsilon^{2}\right)\right) \rightarrow \Gamma(A)\right)$.

Proof. The proof in [BL94] carries over easily to give that statement (a) implies statement (b). For the reverse direction, we adapt the arguments of [BL94] as follows.

Let $m: \Gamma \times{ }_{S} \mathcal{Q} \rightarrow \mathcal{Q}$ be the group action and $\mathrm{pr}_{2}: \Gamma \times{ }_{S} \mathcal{Q} \rightarrow \mathcal{Q}$ the projection. We also have a given isomorphism $\phi: m^{*} \mathcal{L} \rightarrow \operatorname{pr}_{2}^{*} \mathcal{L}$. Let $\sigma=\phi\left(m^{*} s\right)-\operatorname{pr}_{2}^{*} s$. Assume statement (b); to prove statement (a), we need to show $\sigma=0$.

Suppose $\sigma \neq 0$. Write $\Gamma$ and $\mathcal{Q}$ as ind-schemes over $S$ :

$$
\Gamma=\lim _{\rightarrow} \Gamma^{(N)}, \quad \mathcal{Q}=\lim _{\rightarrow} \mathcal{Q}^{(M)} .
$$

Here, $M$ and $N$ vary over the natural numbers, and $\Gamma^{(N)}$ and $\mathcal{Q}^{(M)}$ are schemes of finite type over $S$.

Assume that $\sigma$ is non-zero on $\Gamma^{\left(N_{0}\right)} \times_{S} \mathcal{Q}^{\left(M_{0}\right)}$. Base changing to $T=Q^{\left(M_{0}\right)}$, we have an ind-scheme $\Gamma_{T}=\lim _{\rightarrow} \Gamma^{(N)} \times_{S} T=\lim _{\rightarrow} \Gamma_{T}^{(N)}$. The restriction of $\sigma$ to $\Gamma_{T}$ is clearly non-zero.

Let $x \in \Gamma_{T}^{\left(N_{0}\right)}$ be a closed point of $\Gamma_{T}$ such that $\sigma$ is non-zero in the stalk at $x$ in $\Gamma_{T}^{\left(N_{0}\right)}$. Let $y \in T$ be the image. For every positive integer $u$, we can form the base change diagram

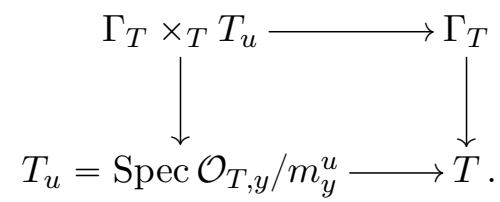

Let $u \geqslant 0$ be the smallest integer such that the image of $\sigma$ is non-zero in $\Gamma_{T} \times_{T} T_{u+1}$ (since $\sigma$ restricted to some $\Gamma_{T}^{(N)}$ is non-zero, such a $u$ exists).

Now, there is a map

$$
H^{0}\left(\left(\Gamma_{T}\right)_{y}, \mathcal{O}_{\left(\Gamma_{T}\right)_{y}}\right) \otimes_{k} \mathcal{L}_{y} \otimes_{k} m_{T, y}^{u} / m_{T, y}^{u+1} \rightarrow H^{0}\left(\Gamma_{T} \times_{T} T_{u+1}, \mathcal{L}\right) .
$$

We claim that this map is injective and $\sigma$ is in the image. The local situation is the following: 


\section{P. Belkale And N. FAKhruddin}

(i) Set $T_{u+1}=\operatorname{Spec}(A)$, where $(A, m)$ is a local ring and $m^{u+1}=0$ and $k=A / m$ (and $A$ is a $k$-algebra).

(ii) Write $\Gamma_{A}$ as an inductive limit of $\operatorname{Spec}\left(B_{j}\right)$, and set $B=\lim _{\longleftarrow} B_{j}$, where the $B_{j}$ are $A$-algebras.

(iii) The rings $B_{j} / m_{j} B_{j}$ are of finite type over $k$.

(iv) There is a section of $\Gamma_{A} \rightarrow \operatorname{Spec}(A)$ passing through any closed point of the central fiber (using the formal smoothness of $\Gamma$ ).

(v) Pick a $k$-basis $a_{1}, \ldots, a_{r}$ of $m^{u}$.

(vi) Suppose $f \in \lim _{j} m^{u} B_{j} \subseteq \lim _{\longleftarrow} B_{j}$.

Our aim is then to show that

- we can write $f$ uniquely as $f=\sum_{i=1}^{r} a_{i} g_{i}$, where $g_{i} \in \underset{\lim }{\longleftarrow} B_{j} / m B_{j}$.

We can find such lifts $g_{i}$ at the level of each $B_{j}$, non-uniquely. The ambiguity is the module of relations

$$
M_{j}=\left\{\left(h_{1}, \ldots, h_{r}\right) \in\left(B_{j} / m B_{j}\right)^{\oplus r} \mid \sum a_{i} h_{i}=0 \in m^{u} B_{j}\right\} .
$$

Therefore, there are exact sequences

$$
0 \rightarrow M_{j} \rightarrow\left(B_{j} / m B_{j}\right)^{\oplus r} \rightarrow m^{u} B_{j} \rightarrow 0 .
$$

It now suffices to show (using Mittag-Leffler properties; see, for example, [Har77, Proposition II.9.1]) that for any $j$, there is an $n>j$ such that $M_{n} \rightarrow M_{j}$ is the zero map. This will also lead to an isomorphism (recall that $m^{u+1}=0$ )

$$
m^{u} \otimes_{k} \lim _{\longleftarrow} B_{i} / m B_{i} \stackrel{\sim}{\rightarrow} \underset{i}{\lim } m^{u} B_{i} \subseteq \lim _{\leftarrow} B_{i} .
$$

First, we show that there is an $\ell>j$ such that any nilpotent element in $B_{\ell} / m B_{\ell}$ goes to zero in $B_{j} / m B_{j}$. This is because the closed fiber of $\Gamma_{A}$ is the limit of an inductive system $Y_{c}$ of reduced varieties. Therefore, there exists an $\ell>j$ such that we have a factoring $\operatorname{Spec}\left(B_{j} / m B_{j}\right) \rightarrow Y_{c} \rightarrow$ $\operatorname{Spec}\left(B_{\ell} / m B_{\ell}\right)$. Any nilpotent element in $B_{\ell} / m B_{\ell}$ must vanish on $Y_{c}$, so goes to zero in $B_{j} / m B_{j}$.

Fix such an $\ell$, and let $Z_{\ell}=\operatorname{Spec}\left(B_{\ell} / m B_{\ell}\right)$. The scheme $Z_{\ell} \times_{k} \operatorname{Spec}(A)$ has a map to $\Gamma$ (over $\operatorname{Spec}(A))$ lifting the map of $Z_{\ell}$ to the closed fiber of $\Gamma_{A}$ (using formal smoothness). Pick an $n$ such that $Z_{\ell} \times_{k} \operatorname{Spec}(A) \rightarrow \Gamma$ factors through $\operatorname{Spec}\left(B_{n}\right)$. This $n$ "works": Given an equation $\sum a_{i} h_{i}=0 \in m^{u} B_{n}$ with $h_{i} \in B_{n} / m B_{n}$, we can restrict to the $\operatorname{sections}$ of $\operatorname{Spec}\left(B_{n}\right) \rightarrow \operatorname{Spec}(A)$ coming from the map $Z_{\ell} \times{ }_{k} \operatorname{Spec}(A) \rightarrow \operatorname{Spec}\left(B_{n}\right)$. Since $a_{1}, \ldots, a_{r}$ are $k$-linearly independent, the $h_{i}$ vanish at all points in the image of $Z_{\ell}^{5}$ (that is, give a nilpotent function on $Z_{\ell}$ ), and hence map to $0 \in B_{j} / m B_{j}$, as desired.

Therefore, we get a non-zero

$$
\bar{\sigma} \in H^{0}\left(\left(\Gamma_{T}\right)_{y}, \mathcal{O}_{\left(\Gamma_{T}\right)_{y}}\right) \otimes_{k} V, \quad V=\mathcal{L}_{y} \otimes_{k} m_{T, y}^{u} / m_{T, y}^{u+1} .
$$

(We define $m_{T, y}^{0}$ to be the local ring of $T$ at $y$.)

Now, $\bar{\sigma}$ is a section of the trivial vector bundle on $\left(\Gamma_{T}\right)_{y}$ with fibers $V$, and it suffices to show that $\bar{\sigma}$ is constant (that is, given by an element of $V$ ): the constant has to be zero since $\sigma$ is zero when pulled back via the identity section $\mathcal{Q} \rightarrow \Gamma \times{ }_{S} \mathcal{Q}$.

Let $A=\mathcal{O}_{T, y} / m_{T, y}^{u+1}$. Assume that $\bar{\sigma}$ is not zero on the integral ind-scheme $\left(\Gamma_{T}\right)_{y}$. Write $V=k^{r}$ for some $r$. Then, there is a tangent vector $X: \operatorname{Spec}\left(k[\epsilon] /\left(\epsilon^{2}\right)\right) \rightarrow\left(\Gamma_{T}\right)_{y}$ which does

${ }^{5}$ Write $h_{i}$ as the sum of a constant and a function which vanishes at a given point of the closed fiber to facilitate the pull back to $\operatorname{Spec}(A)$ via sections. 


\section{PRINCIPAL BUNDLES ON SINGULAR CURVES}

not kill one of the $r$ components of $\bar{\sigma}$. Since $\Gamma_{T}$ is formally smooth over $T$, we can extend $X$ to a tangent $X_{A}: \operatorname{Spec}\left(A[\epsilon] / \epsilon^{2}\right) \rightarrow \Gamma_{T}$ over $T$. Now, $X_{A}$ gives rise to a map $Y_{A}: \operatorname{Spec}(A) \rightarrow \Gamma_{T}$ obtained as a composition

$$
\operatorname{Spec}(A) \rightarrow \operatorname{Spec}\left(A[\epsilon] / \epsilon^{2}\right) \stackrel{X_{A}}{\longrightarrow} \Gamma_{T} .
$$

Set $X_{A}^{\prime}$ to be the composition

$$
\operatorname{Spec}\left(A[\epsilon] / \epsilon^{2}\right) \rightarrow \operatorname{Spec}(A) \stackrel{Y_{A}}{\longrightarrow} \Gamma_{T} .
$$

We have a tautologous $A$-point $p$ of $\mathcal{Q}_{A}$. Let $q$ denote the point $p$ translated by $Y_{A}$. Consider the two elements in $\mathcal{L}_{p} \otimes_{A} A[\epsilon] /\left(\epsilon^{2}\right)$ obtained by pulling back $\sigma$ under $X_{A}$ and $X_{A}^{\prime}$, respectively. By our assumption, the difference $\epsilon \Delta$ of these elements is non-zero. We therefore get a non-zero element $\Delta \in \mathcal{L}_{p}$.

Set $\alpha=X_{A} \circ\left(X_{A}^{\prime}\right)^{-1} \in \operatorname{Lie}(\Gamma)(A)$; we obtain $\alpha \sigma_{A} \in H^{0}\left(\mathcal{Q}_{A}, \mathcal{L}_{A}\right)$. It is easy to see that $\Delta \in \mathcal{L}_{p}$ agrees with the value of $\alpha \sigma_{A}$ at $q$ (an element of $\mathcal{L}_{q}$ ) under the isomorphism of $A$-modules $\mathcal{L}_{q} \stackrel{\sim}{\rightarrow} \mathcal{L}_{q}$ given by $X_{A}^{\prime}$.

Since by assumption (b), we have $\alpha \sigma_{A}=0$ and $\Delta \neq 0$, we reach a contradiction.

Proposition 6.12. Suppose that $\Gamma \rightarrow S=\operatorname{Spec}(R)$ has integral fibers, where $S$ is a scheme of finite type over $k=\mathbb{C}$. Let $\phi: \Gamma \rightarrow \operatorname{Aut}(V)$, where $V$ is a finite-dimensional vector bundle over $S$, be a map of $R$-groups. Assume that for every $R$-algebra $A$, the group $\operatorname{Lie}(\Gamma)(A)=$ $\operatorname{ker}\left(\Gamma\left(A[\epsilon] / \epsilon^{2}\right) \rightarrow \Gamma(A)\right)$ maps to $0 \in \operatorname{End}(V) \otimes_{R} A$ under $\phi$. Then $\phi$ is the trivial morphism.

Proof. We may assume, by passing to an affine cover of $S$, that $V$ is trivial. Now, assume that $\phi$ is not trivial, and let $y \in S$ and $u \geqslant 0$ be such that the image of $\phi$ is not the identity in $\Gamma \times{ }_{S} S_{u+1}$, where $S_{u+1}=\operatorname{Spec} \mathcal{O}_{S, y} / m_{y}^{u+1}$, with $u$ the smallest possible. Base changing the picture to $S_{u}$, we get a matrix of functions $\left(f_{a, b}\right)$ on $\Gamma$. Therefore, $\left(f_{a, b}\right)$ differs from the identity matrix by a matrix $G=\left(g_{a b}\right)$ with $g_{a, b} \in m^{u} B_{j}$ for every $j$, where we have expressed $\Gamma_{S_{u+1}}$ as $\lim _{\longleftarrow} B_{j}$.

The matrix $G$ can be lifted to a matrix with coefficients in $\lim _{\longleftarrow} m^{u} \otimes_{k} B_{j} / m B_{j}$ (as before, see (6.9)), and our aim is to show that elements of $\lim _{\longleftarrow} B_{j} / m B_{j}$ coming from matrix coefficients (after choosing a basis of $m^{u}$ ) have derivatives zero. The rest of the proof is similar to that of Proposition 6.11, using the group action to move sections of $\Gamma$ to the identity section.

\section{Picard groups}

Fix an $n$-pointed curve $(C ; \vec{p})$ with arbitrary singularities. Let $m$ be the number of irreducible components of $C$. Choose smooth points $q_{1}, \ldots, q_{m} \in C$ distinct from $p_{1}, \ldots, p_{n}$ and local uniformizing parameters at the points $p_{1}, \ldots, p_{n}$.

We have a morphism of stacks $\mathcal{Q}_{G}^{m} \times(G / B)^{n} \rightarrow \operatorname{Parbun}_{G}(C ; \vec{p})$, which by Proposition 5.2 induces an isomorphism

$$
L_{C-\left\{q_{1}, \ldots, q_{m}\right\}}(G) \backslash \mathcal{Q}_{G}^{m} \times(G / B)^{n} \rightarrow \operatorname{Parbun}_{G}(C ; \vec{p}) .
$$

Recall that $\operatorname{Pic} \mathcal{Q}_{G}=\mathbb{Z}$.

Proposition 7.1. The natural pull-back morphism

$$
\operatorname{Pic}\left(\operatorname{Parbun}_{G}(C ; \vec{p})\right) \rightarrow \operatorname{Pic}\left(\mathcal{Q}_{G}^{m} \times(G / B)^{n}\right)=\mathbb{Z}^{m} \oplus \oplus_{i=1}^{n} \operatorname{Pic}(G / B)
$$

is an isomorphism. 


\section{P. BELKAle AND N. FAKHRUdDin}

Proof. By Proposition 5.2, $\operatorname{Pic}\left(\operatorname{Parbun}_{G}(C ; \vec{p})\right)$ coincides with the group of $L_{C-\left\{q_{1}, \ldots, q_{m}\right\}}(G)$ linearized bundles on $\mathcal{Q}_{G}^{m} \times(G / B)^{n}$. By Proposition 5.3 and [LS97, Corollary 5.1], there can be at most one such linearization of a line bundle on $\mathcal{Q}_{G}^{m} \times(G / B)^{n}$. This shows that the morphism (7.1) is injective.

To show the surjectivity, we use the line bundles of the form $E \mapsto E_{p_{i}} \times_{B} \mathbb{C}$, where $B$ acts on $\mathbb{C}$ via a character $\chi$ to produce line bundles of the form $\left(0^{m}, 0, \ldots, \chi_{i}, \ldots, 0\right)$ with $\chi$ in the $i$ th place. This takes care of all the $G / B$ contributions. For the remaining contributions, we use diagrams induced by restricting bundles to irreducible components. If $q_{j}$ is on the irreducible component $C_{j}$, consider

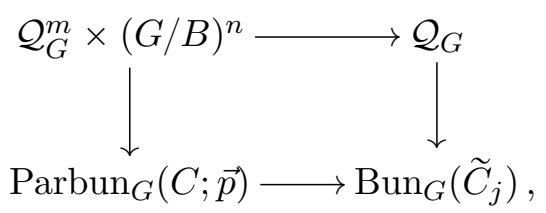

where the horizontal map on top is the projection to the $j$ th factor of $\mathcal{Q}_{G}^{m}$ and $\widetilde{C}_{j}$ the normalization of $C_{j}$. Here, we use the fact that the uniformization map $\mathcal{Q}_{G} \rightarrow \operatorname{Bun}_{G}\left(\widetilde{C}_{j}\right)$ corresponding to $\left(\widetilde{C}_{j}, q_{j}\right)$ is an isomorphism on Picard groups by [Las98, Sor99].

Let $G \rightarrow \mathrm{SL}(V)$ be an irreducible representation of $G$. Associated to $V$, there is a notion of a Dynkin index $d_{V}$ (cf. [KNR94]). It has the following property: if $p$ is smooth point (with local coordinates) on a smooth projective curve $C$, the associated morphism $\mathcal{Q}_{G} \rightarrow \operatorname{Bun}_{G}(C)$ pulls back the determinant of the cohomology line bundle $\operatorname{det}\left(H^{*}\left(C, E \times_{P} V\right)\right)(\operatorname{see}(6.7))$ on $\operatorname{Bun}_{G}(C)$ to $d_{V}$ times the positive generator $\mathcal{O}(1)$ of the Picard group of $\mathcal{Q}_{G}$ (see [LS97]).

Lemma 7.2. Suppose that $y_{1}, \ldots, y_{m}$ are distinct smooth points on a possibly singular projective curve $C$. The corresponding map (after choosing local coordinates at the points $p_{i}$ ) $\mathcal{Q}_{G}^{m} \rightarrow$ $\operatorname{Bun}_{G}(C)$ pulls back the determinant of cohomology bundle $\operatorname{det}\left(H^{*}\left(C, E \times_{P} V\right)\right)$ to $\bigotimes_{i=1}^{m} \mathcal{O}\left(d_{V}\right)$.

Proof. Since the Picard group of $\mathcal{Q}_{G}$ is $\mathbb{Z}$, it follows that any line bundle on $\mathcal{Q}_{G}^{m}$ is of the form $\bigotimes_{i=1}^{m} \mathcal{O}\left(a_{i}\right)$. It therefore suffices to assume $m=1$. Let $\pi: \widetilde{C} \rightarrow C$ be the normalization of $C$; we have maps $\mathcal{Q}_{G} \rightarrow \operatorname{Bun}_{G}(C) \rightarrow \operatorname{Bun}_{G}(\widetilde{C})$. The statement then comes down to verifying the canonical equality $\operatorname{det}\left(H^{*}\left(\widetilde{C}, \pi^{*} E \times{ }_{P} V\right)\right)=\operatorname{det}\left(H^{*}\left(C, E \times{ }_{P} V\right)\right)$ for any principal bundle $E$ on $C$. (Note that $\mathcal{Q}_{G} \rightarrow \operatorname{Bun}_{G}(\widetilde{C})$ produces $G$-bundles on $\widetilde{C}$ which are trivial on connected components of $\widetilde{C}$ other than the one mapped to by $y_{1}$, so we may assume that $\widetilde{C}$ is connected.)

We show that if $W$ is a vector bundle with trivial determinant on $C$, then $\operatorname{det}\left(H^{*}\left(C, \pi_{*} \pi^{*} W\right)\right)$ $=\operatorname{det}\left(H^{*}(C, W)\right)$. Let $M=\pi_{*} \mathcal{O} / \mathcal{O}$. We are reduced to showing that $\operatorname{det}\left(H s^{*}(C, M \otimes W)\right)$ is trivial. But $M$ is torsion on $C$ and can be filtered in such a way that the graded quotients are (directs sums) of the form $i_{*} k$, where $i$ is the inclusion of a closed point in $C$. The case $M=i_{*} k$ is immediate since $W$ has trivial determinant.

\section{ACKNOWLEDGEMENTS}

We thank Yogish Holla for useful discussions and the referee for some helpful comments. This work was begun when P. B. was visiting TIFR Mumbai in July-August 2015. P. B. thanks N. F. for the invitation and TIFR for its hospitality. 


\section{PRINCIPAL BUNDLES ON SINGULAR CURVES}

\section{REFERENCES}

AG60 M. Auslander and O. Goldman, The Brauer group of a commutative ring, Trans. Amer. Math. Soc. 97 (1960), 367-409; doi:10.2307/1993378.

Art69 M. Artin, Algebraization of formal moduli. I, in Global Analysis (Papers in Honor of K. Kodaira) (University Tokyo Press, Tokyo, 1969), 21-71.

Ati57 M. F. Atiyah, Vector bundles over an elliptic curve, Proc. London Math. Soc. (3) 7 (1957), no. 1, 414-452; doi:10.1112/plms/s3-7.1.414.

BG18 P. Belkale and A. Gibney, On finite generation of the section ring of the determinant of cohomology line bundle, Trans. Amer. Math. Soc., published online on 14 December 2018, doi:10.1090/tran/7564, to appear in print.

Bia70 A. Białynicki-Birula, Rationally trivial homogeneous principal fibrations of schemes, Invent. Math. 11 (1970), no. 3, 259-262; doi:10.1007/BF01404652.

BL94 A. Beauville and Y. Laszlo, Conformal blocks and generalized theta functions, Comm. Math. Phys. 164 (1994), no. 2, 385-419; doi:10.1007/BF02101707.

BL95_ Un lemme de descente, C. R. Acad. Sci. Paris Sér. I Math. 320 (1995), no. 3, 335-340.

BLR90 S. Bosch, W. Lütkebohmert and M. Raynaud, Néron models, Ergeb. Math. Grenzgeb. (3), vol. 21 (Springer-Verlag, Berlin, 1990); doi:10.1007/978-3-642-51438-8.

BP95 E. Bayer-Fluckiger and R. Parimala, Galois cohomology of the classical groups over fields of cohomological dimension $\leqslant$ 2, Invent. Math. 122 (1995), no. 2, 195-229; doi:10.1007/BF01231443.

BS68 A. Borel and T. A. Springer, Rationality properties of linear algebraic groups. II, Tôhoku Math. J. 20 (1968), no. 4, 443-497; doi:10.2748/tmj/1178243073.

Con14 B. Conrad, Reductive group schemes, in Autour des schémas en groupes, Vol. I, Panor. Synthèses, vol. 42/43 (Soc. Math. France, Paris, 2014) 93-444.

dJo05 A. J. de Jong, A result of Gabber, 2005, available at http://www. math.columbia.edu/ dejong/ papers/2-gabber.pdf.

dJHS11 A.J. de Jong, X. He and J. M. Starr, Families of rationally simply connected varieties over surfaces and torsors for semisimple groups, Publ. Math. Inst. Hautes Études Sci. (2011), no. 114, 1-8; doi:10.1007/s10240-011-0035-1.

Dri06 V. Drinfeld, Infinite-dimensional vector bundles in algebraic geometry: an introduction, in The Unity of Mathematics, Progr. Math., vol. 244 (Birkhäuser Boston, Boston, MA, 2006), 263-304; doi:10.1007/0-8176-4467-9_7.

DS95 V.G. Drinfeld and C. Simpson, B-structures on G-bundles and local triviality, Math. Res. Lett. 2 (1995), no. 6, 823-829; doi:10.4310/MRL.1995.v2.n6.a13.

Fak12 N. Fakhruddin, Chern classes of conformal blocks, in Compact Moduli Spaces and Vector Bundles, Contemp. Math., vol. 564 (Amer. Math. Soc., Providence, RI, 2012), 145-176; doi:10.1090/conm/564/11148.

Fal94 G. Faltings, A proof for the Verlinde formula, J. Algebraic Geom. 3 (1994), no. 2, 347-374.

FS13 M. Fedorchuk and D. I. Smyth, Alternate compactifications of moduli spaces of curves, in Handbook of Moduli, Vol. I, Adv. Lect. Math., vol. 24 (Int. Press, Somerville, MA, 2013), 331-413.

Gab81 O. Gabber, Some theorems on Azumaya algebras, The Brauer Group (Sem., Les Plans-surBex, 1980), Lecture Notes in Math., vol. 844 (Springer, Berlin - New York, 1981), 129-209; doi:10.1007/BFb0090480.

Gro68 A. Grothendieck, Le groupe de Brauer. II. Théorie cohomologique, in Dix exposés sur la cohomologie des schémas, Adv. Stud. Pure Math., vol. 3 (North-Holland, Amsterdam, 1968), 67-87.

Har67 G. Harder, Halbeinfache Gruppenschemata über Dedekindringen, Invent. Math. 4 (1967), no. 3, 165-191; doi:10.1007/BF01425754.

Har75 G. Harder, Über die Galoiskohomologie halbeinfacher algebraischer Gruppen. III, J. reine angew. Math. 1975 (1975), no. 274-275, 125-138; doi:10.1515/crll.1975.274-275.125. 


\section{P. Belkale And N. FAKhruddin}

Har77 R. Hartshorne, Algebraic geometry, Grad. Texts in Math., vol. 52 (Springer-Verlag, New York Heidelberg, 1977); doi:10.1007/978-1-4757-3849-0.

Hoo82 R. T. Hoobler, When is $\operatorname{Br}(X)=\operatorname{Br}^{\prime}(X)$ ?, Brauer Groups in Ring Theory and Algebraic Geometry (Wilrijk, 1981), Lecture Notes in Math., vol. 917 (Springer, Berlin - New York, 1982), 231-244.

IM65 N. Iwahori and H. Matsumoto, On some Bruhat decomposition and the structure of the Hecke rings of p-adic Chevalley groups, Publ. Math. Inst. Hautes Études Sci. (1965), no. 25, 5-48; doi:10.1007/BF02684396.

KNR94 S. Kumar, M. S. Narasimhan and A. Ramanathan, Infinite Grassmannians and moduli spaces of G-bundles, Math. Ann. 300 (1994), no. 1, 41-75; doi:10.1007/BF01450475.

Kum87 S. Kumar, Demazure character formula in arbitrary Kac-Moody setting, Invent. Math. 89 (1987), no. 2, 395-423; doi:10.1007/BF01389086.

Las98 Y. Laszlo, Hitchin's and WZW connections are the same, J. Differential Geom. 49 (1998), no. 3, 547-576; doi:10.4310/jdg/1214461110.

Laz64 D. Lazard, Sur les modules plats, C. R. Acad. Sci. Paris 258 (1964), 6313-6316.

LM00 G. Laumon and L. Moret-Bailly, Champs algébriques, Ergeb. Math. Grenzgeb. (3), vol. 39 (Springer-Verlag, Berlin, 2000).

LS97 Y. Laszlo and C. Sorger, The line bundles on the moduli of parabolic G-bundles over curves and their sections, Ann. Sci. École Norm. Sup. (4) 30 (1997), no. 4, 499-525; doi:10.1016/S0012-9593(97)89929-6.

Mat88 O. Mathieu, Formules de caractères pour les algèbres de Kac-Moody générales, Astérisque 159160 (Soc. Math. France, Paris, 1988), 1-267.

Ols07 M. Olsson, Sheaves on Artin stacks, J. reine angew. Math. 2007 (2007), no. 603, 55-112; doi:10.1515/CRELLE.2007.012.

PR08 G. Pappas and M. Rapoport, Twisted loop groups and their affine flag varieties (with an appendix by T. Haines and M. Rapoport, Adv. Math. 219 (2008), no. 1, 118-198; doi:10.1016/j.aim.2008.04.006.

PSV15 I. Panin, A. Stavrova and N. Vavilov, On Grothendieck-Serre's conjecture concerning principal G-bundles over reductive group schemes: I, Compos. Math. 151 (2015), no. 3, 535-567; doi:10.1112/S0010437X14007635.

Ser58 J.-P. Serre, Modules projectifs et espaces fibrés à fibre vectorielle, Séminaire P. Dubreil, Algèbre et théorie des nombres, tome 11, no. 2, Exposé 23, 1957-1958 (Secrétariat math., Paris, 1958), $1-18$.

Sha82 I. R. Shafarevich, On some infinite-dimensional groups. II, Math. USSR Izv. 18 (1982), no. 1, 185-194; doi:10.1070/IM1982v018n01ABEH001379.

Sor96 C. Sorger, La formule de Verlinde, Sémin. Bourbaki (1994/95), Exp. No. 794, Astérisque 237 (Soc. Math. France, Paris, 1996), 87-114.

Sor99 On moduli of $G$-bundles of a curve for exceptional $G$, Ann. Sci. École Norm. Sup. (4) 32 (1999), no. 1, 127-133; doi:10.1016/S0012-9593(99)80011-1.

Sor00_, Lectures on moduli of principal G-bundles over algebraic curves, School on Algebraic Geometry (Trieste, 1999), ICTP Lect. Notes, vol. 1 (Abdus Salam Int. Cent. Theoret. Phys., Trieste, 2000), 1-57.

SSV12 A. Smolensky, B. Sury and N. Vavilov, Gauss decomposition for Chevalley groups, revisited, Int. J. Group Theory 1 (2012), no. 1, 3-16; doi:10.22108/ijgt.2012.467.

Ste73 M. R. Stein, Surjective stability in dimension 0 for $K_{2}$ and related functors, Trans. Amer. Math. Soc. 178 (1973), 165-191; doi:10.2307/1996696.

Ste65 R. Steinberg, Regular elements of semisimple algebraic groups, Publ. Math. Inst. Hautes Études Sci. (1965), no. 25, 49-80; doi:10.1007/BF02684397. 


\section{PRINCIPAL BUNDLES ON SINGULAR CURVES}

Ste68_, Lectures on Chevalley groups, Univ. Lecture Ser., vol 66 (Amer. Math. Soc, 2016); doi:10.1090/ulect/066

TUY89 A. Tsuchiya, K. Ueno and Y. Yamada, Conformal field theory on universal family of stable curves with gauge symmetries, in Integrable Systems in Quantum Field Theory and Statistical Mechanics, Adv. Stud. Pure Math., vol. 19 (Academic Press, Boston, MA, 1989), 459-566.

Wan11 J. Wang, The moduli stack of G-bundles, 2011, arXiv:1104.4828.

Prakash Belkale belkale@email.unc.edu

Department of Mathematics, University of North Carolina, Chapel Hill, NC 27599, USA

Najmuddin Fakhruddin naf@math.tifr.res.in

School of Mathematics, Tata Institute of Fundamental Research, Homi Bhabha Road, Mumbai 400005, India 